\title{
中新生代两类极热事件的环境变化、生态效应与 驱动机制
}

\author{
胡修棉”, 李娟, 韩中, 李永祥
}

南京大学内生金属矿床成矿机制研究国家重点实验室, 地球科学与工程学院, 南京 210023

*通讯作者, E-mail: huxm@nju.edu.cn

收稿日期：2019-08-20; 收修改稿日期：2020-02-24; 接受日期：2020-03-30; 网络版发表日期：2020-05-19 国家自然科学基金基础中心项目(批准号: 41888101)和国家杰出青年科学基金项目(批准号: 41525007)资助

摘要 对地质历史时期发生的极热事件进行深入分析可为认识和应对现今全球气候变暖提供借鉴与依据. 根据 碳同位素偏移特征，可以将中新生代五次典型的极热事件划分为碳同位素负偏移类和碳同位素正偏移类两类。第 一类极热事件以碳同位素总体负偏移为特征，以二叠纪-三叠纪界线事件(PTB， 252Ma)、早侏罗世Toarcian早期 大洋缺氧事件(TOAE, 183Ma)、古新世-始新世界线极热事件(PETM, 56Ma)为代表. 第二类极热事件以碳同位 素总体正偏移为特征, 以白严纪Aptian早期大洋缺氧事件(OAE1a, 120Ma)、白严纪Cenomanian末期大洋缺氧事 件(OAE2， ９4Ma)为代表。碳同位素负偏移定义的极热事件造成温度、沉积作用、生物多样性等发生明显的变 化，陆地环境出现野火频发、极端千旱、酸雨、臭氧层被破坏、金属中毒(如承)和陆地水系变化等，海洋环境出 现碳酸盐台地消亡、大洋酸化、大洋缺氧等现象, 全球陆地和海洋生物尤其是浅海生物发生不同程度的绝灭. 碳 同位素正偏移定义的极热事件造成海水快速增温和大洋广泛缺氧, 有机质大规模埋藏, 出现黑色页岩沉积, 远洋 生物受到明显的影响(如出现钙质超微危机), 而浅海和陆地生物受到影响不大. 文章提出, 碳同位素负偏移类极热 事件可能为大陆环境下大火成岩省喷发触发, 导致轻碳大规模释放到大气一海洋系统使得全球快速增温，从而引 起一系列环境和生态响应。而碳同位素正偏移类极热事件为深海环境下大火成岩省喷发触发, 把热量和营养物质 直接释放到深海系统, 由于海水缓冲作用导致海洋系统增温明显, 而浅海和陆地系统影响相对较小. 研究结果丰 富了对地质历史时期极热事件的研究，不仅对于理解极热事件、大火成岩省、海洋-陆地环境变化、生物绝灭等 具有重要的意义, 而且为判别极热事件的类型并推断其驱动机制提供了一个新手段.

关键词极热事件, 古气候, 古海洋, 沉积记录, 大火成岩省, 中新生代, 深时

\section{1 概述}

2018年10月政府间气候变化专门委员会(IPCC)发 布的“ $1.5^{\circ} \mathrm{C}$ 全球变暖”报告显示，相比工业革命 1850 年
之前, 地球年平均温度增加了 $(1 \pm 0.2)^{\circ} \mathrm{C}$, 并且以每十年 $0.2^{\circ} \mathrm{C}$ 的速率持续增温(IPCC, 2018), 而最近 30 年对地 球表层大气和海洋温度的持续观测亦证实全球气候正 在变暖. 人类活动(主要是化石燃料的燃烧)导致的温 
室气体(主要是 $\mathrm{CO}_{2}$ ) 排放被认为是引起当前全球变暖 的主要因素(Waters等，2016). 如果大气 $\mathrm{CO}_{2}$ 含量以目 前速率持续增加，是否会导致全球气候从现今的以两 极发育冰盖为特征的冰室气候状态进入以两极无冰盖 为特征的温室气候状态? 这些变化又会对生态和环境 造成多大程度的影响? 人类文明的可持续发展迫切需 要对这种持续增温的变化趋势及其引起的生态、环境 效应有更深入的认识.

目前对于预测增温趋势及其可能导致的气候、生 态、环境变化多依赖于气候模拟. 但遗憾的是, 由于对 真实气候系统运行机制的认识不够全面、气候模型的 时空分辨率不高、各种气候参数的设置具有较强的主 观性等，导致不同的气候模型获得的结果存在较大的 差异. 因此，研究地质历史时期类似的变暖事件及其 生态、环境效应，是人类认识当前所面临的全球变暖 趋势的唯一选择. 地质历史时期的气候状态是动态变 化的, 既有“冰室”气候，也有“温室”气候，而每种气候 状态又会出现一系列极端气候事件，如极冷事件、极 热事件等，中新生代发生多次极热事件，其中比较典 型的极热或增温事件包括二叠纪-三叠纪界线事件 (PTB, 252Ma)、早侏罗世Toarcian早期大洋缺氧事件 (TOAE， 183Ma)、白严纪Aptian早期大洋缺氧事件 (OAE1a， 120Ma)、白严纪Cenomanian末期大洋缺氧 事件(OAE2， 94Ma) 和古新世-始新世极热事件 (PETM， 56Ma)等(图1)(Foster等，2018). 本文在系统 总结这五次极热或增温事件的启动持续时间、增温幅 度与增温速率及其引起的环境、生态变化的基础上, 根据碳同位素特征将其划分为两类，分别为碳同位素 负偏移类极热事件和碳同位素正偏移类极热事件; 并 通过系统比较这两类极热事件引起的环境、生态等方 面的异同，提出这两类极热事件的触发机制可能与大 火成岩省喷发的环境密切相关，这为认识和应对当前 全球气候变暖提供了借鉴和依据.

\section{2 中生代-古近纪五次极热事件的碳同位素 特征与分类}

\section{1 五次极热事件的碳同位素特征}

二叠纪 -三叠纪界线事件 (P T B) 发生在距今 $\sim 252 \mathrm{Ma}$ 的二叠纪末期, 是显生宙以来增温幅度最大的 一次极热事件, 增温幅度约为 $8 \sim 10^{\circ} \mathrm{C}$ (Joachimski等,
2012; Sun等, 2012; Shen等, 2019)(表1), 但是也有部分 学者认为增温幅度不超过 $6^{\circ} \mathrm{C}$ (Brand等, 2012; Cui和 Kump，2015). PTB碳同位素出现至少三次先负偏移, 再恢复的波动过程，最大偏移幅度超过 7\%o(Cao 等, 2009).

早侏罗世Toarcian早期大洋缺氧事件(TOAE) 是发 生在距今 $183 \mathrm{Ma}$ 的一次极热事件, 北半球中纬度地区 增温幅度约为7 $10^{\circ} \mathrm{C}$ (Suan等，2008a；Dera等，2009; Korte等，2015)(表1). TOAE碳同位素存在多次次级负 偏移直至到达最值, 之后恢复正常, 负偏移幅度一般为 -3 -8\%o(Kemp等, 2005; Jenkyns, 2010).

白严纪Aptian早期大洋缺氧事件(OAE 1a)又称 Selli事件，是发生在距今 120Ma的一次增温事件，低 纬度地区和中高纬度海水表层分别增温 5 6和 $2 \sim 4^{\circ} \mathrm{C}$ (Mutterlose等, 2010, 2014; Naafs和Pancost, 2016)(表1). 碳同位素先短暂负偏移后正偏移, 之后再恢复, 正偏移 幅度为2 5\%o(Menegatti等, 1998).

白严纪Cenomanian末期(OAE2)又称Bonarelli事 件, 是发生在距今 $94 \mathrm{Ma}$ 的一次增温事件. 低纬度地区 海水表层增温幅度 $2 \sim 3^{\circ} \mathrm{C}$, 中纬度地区增温 $4 \sim 5^{\circ} \mathrm{C}$, 高 纬度地区增温7 10 ${ }^{\circ} \mathrm{C}$ (Jenkyns等, 2004; Forster等, 2007; Huber等，2018)(表1). 碳同位素总体为正偏移， 但在正偏移之前出现微弱负偏移(Li Y X等, 2017), 正 偏移幅度一般大于 $2 \%$ (Jenkyns, 2010).

古新世-始新世极热事件(PETM)是发生在古新世始新世界线附近( 55.8Ma)的一次极热事件(Kennett和 Stott，1991；Zachos等，2001), 全球平均温度增加约 $4 \sim 5^{\circ} \mathrm{C}$ (Jones等, 2013). 低纬度地区平均增温 $3^{\circ} \mathrm{C}$ (Frieling等, 2017), 中高纬度平均增温 5 $8^{\circ} \mathrm{C}$ (Sluijs等, 2006, 2011; Zachos等，2006)(表1)。碳同位素先快速负偏移， 后恢复(Kennett和Stott，1991)，负偏移幅度为2 7\% (McInerney和Wing, 2011).

\section{2 五次极热事件基于碳同位素偏移特征的分类 及阶段划分}

根据碳同位素偏移特征，将这五次极热事件划分 为碳同位素负偏移类极热事件(Negative Carbon isotope excursion Hyperthermal Event, NCHE)和碳同位 素正偏移类极热事件(Positive Carbon isotope excursion Hyperthermal Event, PCHE).

$\mathrm{NCHE}$ 事件以碳同位素总体负偏移为特征，包括 
(a)

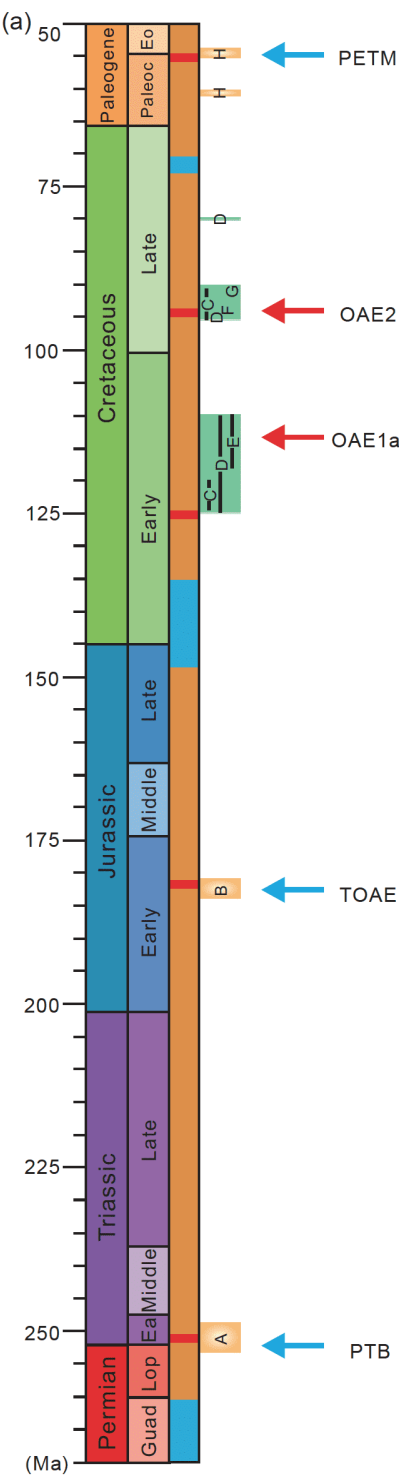

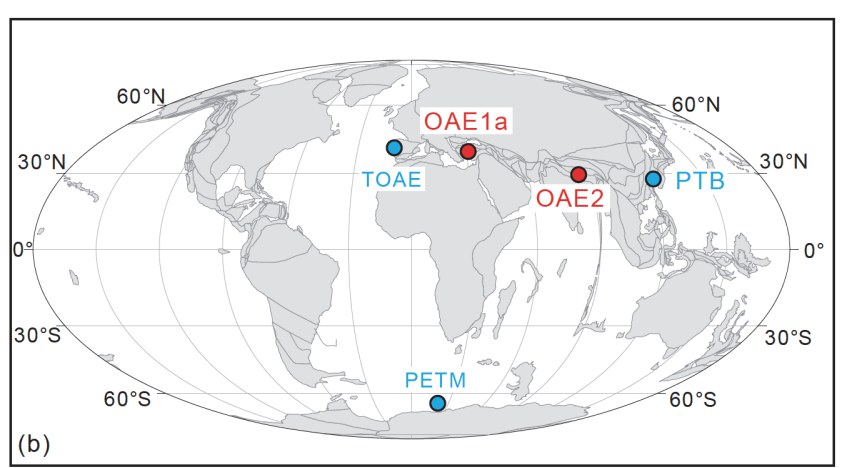
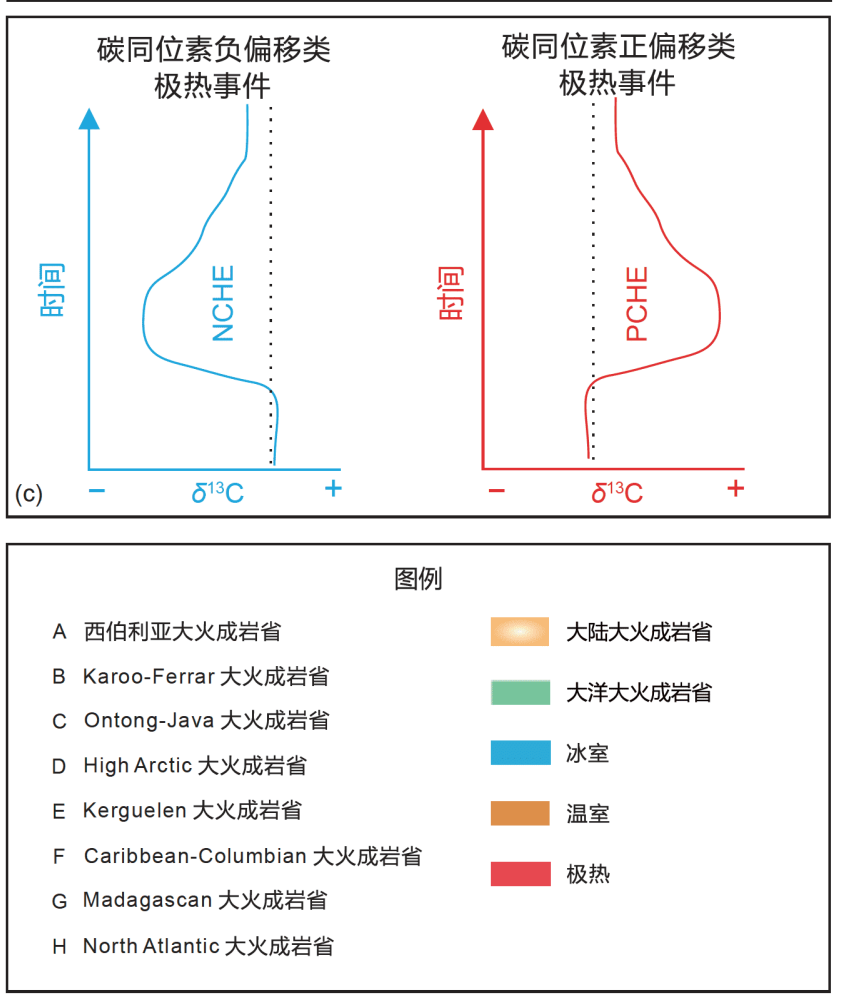

图 1 晚二叠世-古新世地质年代表(a)、金钉子剖面位置(b)和极热事件分类(c)示意图

(a) 地质年代表显示中新生代以来地球气候状态(NRC 2011)、主要极热事件发生时代及其相关的大火成岩省喷发时代. (b) 文中所涉及的五次 极热事件金钉子剖面或代表性剖面的现今地理位置图(www.odsn.de). PTB为中国煤山金钉子剖面(Shen等, 2011; Burgess等, 2014), TOAE为葡 萄牙Peniche金钉子剖面(Hesselbo等, 2007), OAE1a为土耳其Yenicesilar剖面(Hu等, 2012), OAE2为藏南贡扎剖面(Li Y X等, 2017), PETM为 ODP 690钻孔(Bains等, 1999). (c) 基于碳同位素特征的极热事件分类示意图: 碳同位素负偏移类极热事件(NCHE)和碳同位素正偏移类极热事 件(PCHE). 据Ogg等(2016)、Ernst和Youbi(2017)和Percival等(2018)

PTB、TOAE、PETM三次极热事件(图2). 这类极热事 件以碳同位素开始负偏移之前的稳定值作为事件的启 动, 以恢复到负偏移之前的稳定值作为事件的结束. 可 划分为一个(PETM、TOAE)或多个(PTB)碳同位素的 负偏移阶段(启动阶段, Onset, NC2)、稳定负平台阶段 (Core或者Body，NC3)、正偏移恢复阶段(Recovery,
$\mathrm{NC4}$ (图2), 事件之前标注为 NC1，事件之后阶段标注 为NC5. NCHE事件最主要的特征在于其 NC2 NC4阶 段出现显著的负偏移，代表事件的持续时间. 这类事 件通常被认为是由于大量富含轻碳同位素的温室气体 进入到大气-海洋系统引起的.

PCHE事件以碳同位素总体正偏移为特征, 包括 
表 1 NCHE与PCHE两类五次极热事件碳同位素启动阶段偏移幅度(CIE)、持续时间、增温幅度对比

\begin{tabular}{|c|c|c|c|c|c|c|c|}
\hline \multirow{2}{*}{\multicolumn{2}{|c|}{ 极热事件 }} & \multirow{2}{*}{$\begin{array}{c}\text { 启动阶段( } \mathrm{NC2} \\
\text { 或PC2)持续 } \\
\text { 时长(kyr) }\end{array}$} & \multirow{2}{*}{$\begin{array}{c}\text { 启动阶段(NC2 } \\
\text { 或PC2)碳同位素 } \\
\text { 偏移 (CIE, \%o) }\end{array}$} & \multicolumn{3}{|c|}{ 温度增幅 $\left({ }^{\circ} \mathrm{C}\right)$} & \multirow{2}{*}{ 参考文献 } \\
\hline & & & & 低纬度 & 中纬度 & 高纬度 & \\
\hline \multirow[t]{2}{*}{ PCHE } & OAE2 & 50 & $>2$ & $2 \sim 3$ & $4 \sim 5$ & $7 \sim 10$ & $\begin{array}{c}\text { Jenkyns等(2004)、Forster等(2007)、Huber等 } \\
\text { (2018) }\end{array}$ \\
\hline & OAE1a & 75 & $2 \sim 5$ & $5 \sim 6$ & $2 \sim 4$ & $2 \sim 3$ & Mutterlose等(2010)、Naafs和Pancost(2016) \\
\hline \multirow{3}{*}{ NCHE } & PETM & 3 & $-2 \sim-6$ & $3 \sim 5$ & $6 \sim 8$ & $5 \sim 9$ & $\begin{array}{c}\text { Thomas等(2002)、Zachos等(2003, 2006)、Aze等 } \\
\text { (2014)、Sluijs等 }(2011,2014)\end{array}$ \\
\hline & TOAE & 150 & $-3 \sim-8$ & - & $7 \sim 10$ & - & Suan等(2008a)、Dera等(2009)、Korte等(2015) \\
\hline & PTB & 60 & $>-7$ & $8 \sim 10$ & - & - & $\begin{array}{l}\text { Sun等(2012)、Joachimski等(2012)、Burgess等 } \\
\text { (2014)、Chen等(2016) }\end{array}$ \\
\hline
\end{tabular}
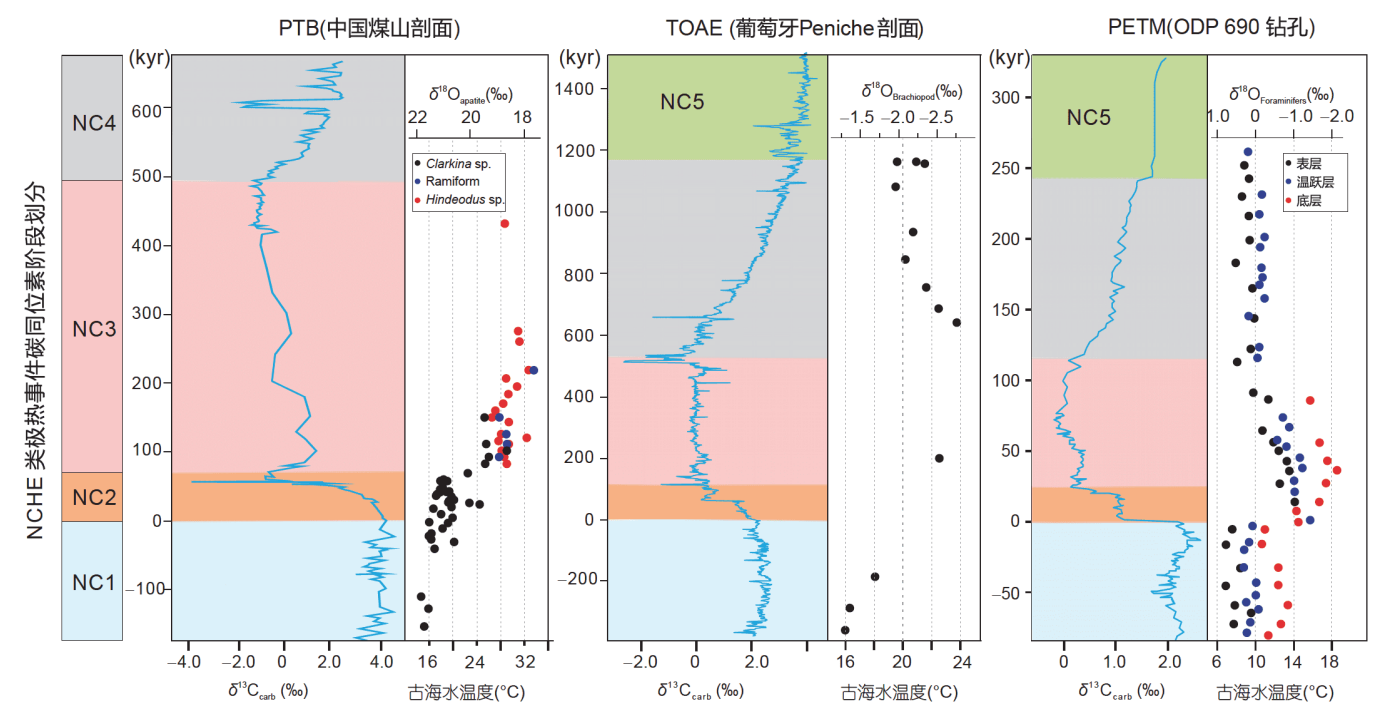

沉积环境和生物变化

图 2 NCHE类极热事件碳同位素及温度变化特征、碳同位素阶段划分、碳同位素各阶段持续时间及其引起的沉积环境和 生物变化

以碳同位素开始负偏移作为事件的启动, 以恢复到负偏移之前的稳定值作为事件的结束. 一般划分为碳同位素的启动( NC2)、持续负偏移(负 平台，NC3)、恢复(NC4)三个阶段，事件之前为NC1，事件之后阶段为NC5. NCHE类极热事件碳同位素、温度数据来源：PTB据Burgess等 (2014), TOAE据Hesselbo等(2007)和Suan等(2010), PETM据Bains等(1999)和Thomas等(2002)

OAE1a和OAE2两次极热事件(图3)。这类极热事件以 碳同位素出现短暂明显(OAE1a)或微弱(OAE2)的负偏 移作为事件的启动, 以恢复到正偏移之前的稳定值作 为事件的结束. 可以划分为四个阶段，包括碳同位素 出现短暂明显(OAE1a)或微弱(OAE2)的负偏移阶段 (Onset，PC2)、正偏移阶段(PC3)、稳定正平台阶段 (Core或者Body，PC4)、负偏移恢复阶段(Recovery, PC5)(图3), 事件之前标注为PC1, 事件之后标注为 PC6. PCHE事件最主要的特征在于PC2段出现短暂负 偏移，很快PC $3 \sim$ P C 5 阶段出现显著的正偏移， PC2 PC5 阶段代表事件的持续时间. 这类事件通常被
认为是由于富含轻碳同位素的温室气体短暂注入大 气-海洋系统中, 很快大洋急剧缺氧引起大规模富含有 机质的黑色页岩埋藏从而导致碳同位素快速正偏移.

\section{3 中生代-古近纪两类极热事件的持续时间}

由于近年来高精度定年技术和旋回地层的应用， 这两类极热事件的持续时间得到了精确限定，为探讨 增温速率提供了时间坐标. 需要提及的是各事件持续 时间通常以凝灰岩层中高精度锆石 $\mathrm{U}-\mathrm{Pb}$ 年龄为锚点， 同时结合旋回地层周期，在同一个旋回周期内采用平 


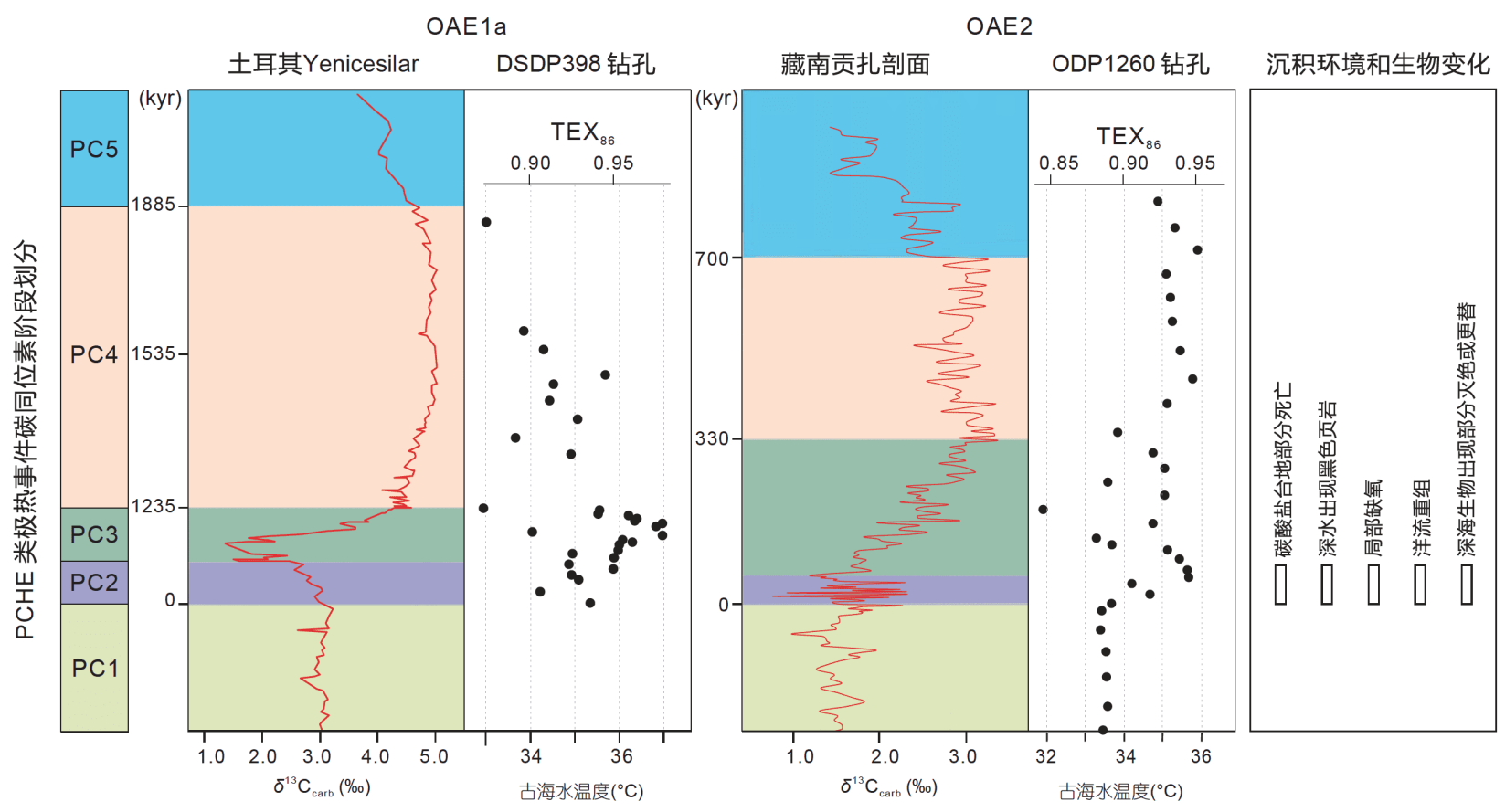

图 3 PCHE类极热事件碳同位素及温度变化特征、碳同位素阶段划分、碳同位素各阶段持续时间及其引起的沉积环境和 生物变化

一般划分为四个阶段包括启动(PC2)、正偏移(PC3)、持续正偏移(正平台, PC4)、恢复(PC5); 事件之前为PC1, 事件之后为PC6. PCHE类极热 事件碳同位素、温度数据来源: OAE1a据Hu等(2012)、Naafs和Pancost(2016), OAE2据Forster等(2007)和Li Y X等(2017)

均沉积速率内插法获得碳同位素偏移阶段的持续时 间. 由于测试精度的限制，误差一般在万年至十万年 左右.

\subsection{NCHE 事件的持续时间}

PTB事件的时限主要基于二叠系-三叠系界线的 金钉子剖面(华南煤山剖面)上凝灰岩层中高精度锆石 U-Pb年龄(Shen等, 2011, 2019; Burgess等, 2014), 结合 碳同位素地层学(Cao等, 2009)获得. PTB事件持续时 间 660kyr(Burgess等, 2014), 其中, 碳同位素负偏移启 动阶段(NC2)持续时间 $58 \mathrm{kyr}$; 碳同位素负平台阶段 (NC3) 持续时间 431 kyr; 碳同位素正偏移恢复阶段 (NC4)持续时间 170kyr(图2).

TOAE事件的持续时间, 目前存在两种不同的观 点: 一种认为其持续时间为 $900 \mathrm{kyr}$, 以葡萄牙Peniche 金钉子剖面旋回地层学获得的研究结果为代表(Suan 等, 2008b; Huang和Hesselbo, 2014), 与德国Dotternhausen、意大利Valdorbia、英国Yorkshire、法国Lorraine (Huang和Hesselbo, 2014; Ruebsam等, 2014)的旋回地 层学和英国Yorkshire的锶同位素地层学McArthur等
(2000)得出的结果相近; 另一种观点认为其持续时间 约为300 500kyr, 以法国巴黎盆地Sancerre-Couy钻孔 和英国Yorkshire剖面的旋回地层学结果为代表 (Kemp 等, 2005, 2011; Boulila等, 2014). 若以葡萄牙Peniche剖 面为准, 则相应的碳同位素负偏移启动阶段( $\mathrm{NC} 2$ )、碳 同位素负平台阶段(NC3)和碳同位素正偏移恢复阶段 (NC4) 的持续时间分别为 150、 450、300 kyr(Suan 等, 2008b)(图2).

PETM事件年龄框架主要通过旋回地层学和 ${ }^{3} \mathrm{He}$ 定年两种方法建立, 目前仍存在比较大的争议. Röhl等 (2003，2007)通过旋回地层学限定PETM持续时间为 150 220ka(图2), Farley和Eltgroth(2003)、Murphy等 (2010) 通过 ${ }^{3} \mathrm{He}$ 定年获得的PETM持续时间为 $120 \sim$ $220 \mathrm{kyr}$ ，而最近Westerhold等(2018)根据ODP钻孔和 Bighorn盆地陆相钻孔资料研究结果, 认为PETM持续 时间为 $200 \mathrm{kyr}$. 在PETM事件启动阶段持续时间上, 一 般认为至少持续了几千年，从8 23kyr不等(Zachos等, 2005; Aziz等, 2008; Charles等, 2011), 然而Wright和 Schaller(2013)根据Millville钻孔所获得的研究结果, 认 为PETM启动时间仅持续了 $13 \mathrm{yr}$, 但这一结果被认为是 
钻探扰动所致，而非原始沉积的韵律(Zeebe等，2014; Pearson和Nicholas, 2014). 关于碳同位素其他各阶段 持续时间，Röhl等(2007)基于ODP1266钻孔的旋回地 层资料, 认为碳同位素负平台阶段 ( $\mathrm{NC} 3$ )持续时间为 $59 \mathrm{kyr}$ ，碳同位素正偏移恢复段( NC4) 持续时间为 113kyr; Aziz等(2008)基于Bighorn盆地陆相记录的旋 回地层学研究结果, 认为碳同位素负平台阶段(NC3) 持续 $115 \mathrm{kyr}$, 而碳同位素正偏移恢复段(NC4)为 $42 \mathrm{kyr}$. Murphy等(2010)在Walvis脊ODP1266 孔通过 ${ }^{3} \mathrm{He}$ 定年 获得碳同位素负平台阶段(NC3)持续时间为 $113 \mathrm{kyr}$, 碳 同位素正偏移恢复段(NC4)可以进一步分为两个阶段: 第一阶段为快速恢复段, 持续时间 $33 \mathrm{kyr}$; 第二个阶段 为缓慢恢复段, 持续时间 $50 \mathrm{kyr}$.

\subsection{PCHE事件的持续时间}

关于OAE1a事件的持续时间, 主要通过旋回地层 学来限定. Menegatti等(1998)根据碳同位素曲线特征, 将OAE1a事件划分为 $\mathrm{C} 2 \sim \mathrm{C} 8$ 阶段, 其中 $\mathrm{C} 3 \sim \mathrm{C} 6$ 阶段持 续时间为 1.0 1.3 Myr(Li等, 2008; Bottini等, 2012). C2 为碳同位素逐步负偏阶段(Socorro等, 2017), C3 为持续 负偏阶段, 本文把这两者一起定义为碳同位素短暂负 偏阶段(PC2), C4 C6 为碳同位素逐步正偏阶段, 定义 为 $\mathrm{PC} 3$ 阶段; $\mathrm{C} 7$ 为碳同位素正平台阶段, 定义为 $\mathrm{PC} 4$ 阶 段; C8 为碳同位素恢复负偏移阶段(PC5)(图3). 在意大 利Gorgo a Cerbara剖面, 若取C3 C6阶段持续时间 $1.11 \mathrm{Myr}$ ，则碳同位素短暂负偏阶段( $\mathrm{PC} 2$ )持续时间为 $70 \mathrm{kyr}$, 碳同位素正偏移阶段(PC3)持续时间为 $1.04 \mathrm{Myr}$, 碳同位素正平台阶段(PC4)持续时间为 $0.75 \mathrm{Myr}$ (Stein 等, 2012; Patruno等，2015; Li等，2016). 在土耳其 Yenicesilar剖面，C2阶段持续时间 $75 \mathrm{kyr}, \mathrm{C} 3$ 持续时间 为 $320 \mathrm{kyr}$ ，则碳同位素短暂负偏阶段 (PC2)持续时间为 $395 \mathrm{kyr}$ (图3); 碳同位素正偏移阶段(PC3)持续时间为 $790 \mathrm{kyr}$ ，碳同位素正平台阶段和负偏移恢复阶段 (PC4 PC5)持续时间为1.3Myr(Hu等, 2012).

关于OAE2事件的持续时间, 主要通过旋回地层学 来限定. 其持续时间争议较大, 从 $200 \mathrm{kyr}$ 到 $>900 \mathrm{kyr}$ 不 等(Sageman等, 2006; Li Y X等, 2017). 在北美西部内 海道(WIS)金钉子剖面, Sageman等(2006)根据有机质 碳同位素旋回地层学限定碳同位素正偏移阶段和碳同 位正平台阶段( $\mathrm{PC} 3 \sim \mathrm{PC} 4)$ 持续时间为 $580 \mathrm{kyr}$, 其中碳 同位素正偏移阶段(PC3)持续时间为 $230 \mathrm{kyr}$, 碳同位
正平台阶段(PC4)持续时间为 $350 \mathrm{kyr}$; 在西藏贡扎剖 面上 $\mathrm{Li} \mathrm{Y} \mathrm{X}$ 等(2017)将 OAE2 的持续时间限定为 $\sim 870 \mathrm{kyr}$, 其中碳同位素微弱负偏移阶段(PC2)、碳同 位素正偏移阶段(PC3)、碳同位正平台阶段 $(\mathrm{PC} 4)$ 、负 偏移恢复阶段(PC5) 持续的时间分别为 $\sim 50$ 、 280 、 370、 170kyr(图3).

\section{4 中生代-古近纪两类极热事件的增温幅度 与速率}

\subsection{NCHE的温度变化}

PTB期间, 在华南煤山剖面上, 牙形石氧同位素数 据指示碳同位素启动阶段(第22 23层)之前古温度为 $\sim 22^{\circ} \mathrm{C}$ (Joachimski等, 2012), 碳同位素启动阶段( NC2) (第24层顶部到26层)温度升高到 $23 \sim 27^{\circ} \mathrm{C}$, 至碳同位素 负平台阶段 $(\mathrm{NC} 3)$ (第 27 层)温度增加到 $29 \sim 30^{\circ} \mathrm{C}$ 或 $33 \sim 35^{\circ} \mathrm{C}$ (不同的牙形石属指示增温幅度不同), 可见在 碳同位素启动阶段(持续 $21 \mathrm{kyr}$ )增温幅度最大, 超过 $8 \sim 10^{\circ} \mathrm{C}$ (Joachimski等, 2012), 与四川上寺剖面和广西 蓬莱滩剖面上增温幅度一致(Cao等, 2009; Joachimski 等, 2012; Shen等, 2019). Sun等(2012)牙形石氧同位素 的研究结果进一步显示, 在碳同位素启动和负平台阶 段温度持续升高, 幅度超过 $14^{\circ} \mathrm{C}$, 至碳同位素负平台 阶段末期(Griesbachian晚期)达到最高值. Chen等 (2016)利用SIMS方法对华南煤山、上寺、代家沟和凉 风垭四个剖面PTB前后牙形石进行了氧同位素分析, 发现快速增温 $\left(\sim 10^{\circ} \mathrm{C}\right)$ 出现在碳同位素启动阶段及生 物大灭绝之后, 与煤山金子剖面第26层相当的层位, 在 灭绝之前及最大灭绝层位 (即煤山24 25层)古海水温 度都无明显变化.

关于TOAE温度的研究主要来自西特提斯洋中纬 度地区, 包括英国、法国、西班牙、葡萄牙等地区, 腕 足、双壳、箭石、鱼骨氧同位素在碳同位素启动阶段 (NC2)出现急剧负偏移, 指示北半球中纬度浅海温度升 高约7 10 C(Dera等, 2009, 2011; Korte等, 2015); 增温 与碳同位素启动阶段同步, 在碳同位素负平台阶段温 度达到最大值, 随后快速降低 $2 \sim 3^{\circ} \mathrm{C}$, 在碳同位素负平 台阶段晚期发生微弱降低或维持较稳定的温度(Dera 等, 2009, 2011; Korte等, 2015).

PETM期间，增温阶段与碳同位素启动阶段 $(\mathrm{NC} 2)$ 同步. 在太平洋赤道地区氧同位素、 $\mathrm{Sr} / \mathrm{Ca}$ 和 $\mathrm{Mg} / \mathrm{Ca}$ 等 
数据指示海水表层温度上升了 $4 \sim 5^{\circ} \mathrm{C}$ (Zachos等, 2003); 低纬度东非坦桑尼亚地区浮游有孔虫氧同位素数据揭 示海水表层温度至少上升了 $3^{\circ} \mathrm{C}$, 最高温度超过 $40^{\circ} \mathrm{C}$ (Aze等, 2014); 在中纬度墨西哥湾地区, 生物标志化 合物为载体 $\left(\mathrm{MBT}-\mathrm{CBT}\right.$ 和 $\left.\mathrm{TEX}_{86}\right)$ 的古温度数据指示海 水表层温度增温幅度为 $6 \sim 8^{\circ} \mathrm{C}$ (Sluijs等, 2014). 中纬度 北大西洋Wilson湖地区 $\mathrm{TEX}_{86}$ 和浮游有孔虫氧同位素 数据指示海水表层温度至少上升了 $8^{\circ} \mathrm{C}$, 最高温度可达 $33^{\circ} \mathrm{C}$ (Zachos等, 2006). 高纬度太平洋西南部ODP 1772 孔 $\mathrm{TEX}_{86}$ 数据指示海水表层温度增温 $\sim 7^{\circ} \mathrm{C}$ (Sluijs等, 2011); 南大洋ODP 690孔有孔虫氧同位素数据表明海 水表层温度升高约 5 9 ${ }^{\circ} \mathrm{C}$ (Thomas等, 2002). 北极地区 IODP 302 孔 $\mathrm{TEX}_{86}$ 数据指示海水表层温度增温 $5^{\circ} \mathrm{C}$ (Sluijs等, 2006). 基于以上数据, 我们可以获得: PETM 期间低纬度地区海水表层温度上升了 $3 \sim 5^{\circ} \mathrm{C}$, 中、高纬 度地区海水表层温度上升了 $5 \sim 9^{\circ} \mathrm{C}$, 显示明显的高纬度 放大效应.

\subsection{PCHE的温度变化}

OAE1a时期，低纬度大西洋地区海水表层温度约 为30 36 ${ }^{\circ} \mathrm{C}$, 在碳同位素正偏移阶段 (PC3)开始时温度 降低 $\sim 4^{\circ} \mathrm{C}$, 随后立刻升高 $\sim 6^{\circ} \mathrm{C}$ (Dumitrescu和Brassell, 2006). 中纬度北大西洋地区 $\mathrm{TEX}_{86}$ 数据表明在OAE1a 碳同位素短暂负偏移阶段( $\mathrm{PC} 2$ )海水表层温度上升约 $2 \sim 4^{\circ} \mathrm{C}$, 而在碳同位素正偏移阶段 $(\mathrm{PC} 3)$ 海水表层温度 下降约 $4 \sim 6^{\circ} \mathrm{C}$ (Naafs 和Pancost, 2016). 在中纬度北半 球, $\mathrm{TEX}_{86}$ 和箭石氧同位素数据指示海水表层温度在 碳同位素短暂负偏移阶段(PC2)升高约 $3 \sim 4^{\circ} \mathrm{C}$ (Mutterlose等, 2010, 2014). 在高纬度南大洋地区, OAE1a期间 海水表层温度在碳同位素短暂负偏移阶段( $\mathrm{PC} 2)$ 上升 约 $2 \sim 3{ }^{\circ} \mathrm{C}$, 之后碳同位素正偏移阶段 $(\mathrm{PC} 3)$ 出现一次变 冷事件, 温度降低 $3^{\circ} \mathrm{C}$ (Jenkyns等, 2012).

OAE2时期, 不同纬度地区海洋表层温度明显升高 (Gustafsson等, 2003; O’Brien等, 2017; Huber等, 2018). 在赤道大西洋地区, 浮游有孔虫氧同位素和 $\mathrm{TEX}_{86}$ 数 据指示海水表层温度上升了约 $2 \sim 3^{\circ} \mathrm{C}$ (Forster等, 2007). 中纬度大西洋地区 $\mathrm{TEX}_{86}$ 数据表明在碳同位素正偏移 阶段(PC3)出现急剧降温约 $4 \sim 5^{\circ} \mathrm{C}$, 然后在碳同位素正 平台阶段(PC4)下部迅速增温约 $4 \sim 5^{\circ} \mathrm{C}$, 海水表层温度 达 $34^{\circ} \mathrm{C}$ (Sinninghe Damsté等, 2010; Huber等, 2018). 北 半球高纬度地区海水表层温度上升约7 $8^{\circ} \mathrm{C}$ (Jenkyns
等, 2004), 而南半球高纬度地区海水表层温度上升 $\sim 10^{\circ} \mathrm{C}$ (Huber等，2018). OAE2期间亦存在短暂的温度 变化事件(Forster等, 2007; O’Brien等, 2017), 其中在欧 洲地区发现一次短暂的变冷事件, 打断了 OAE2 期间整 体增温的趋势(Jenkyns等, 2017). 变冷事件主要归因于 大规模有机质埋藏(Jarvis等，2006)或增强的大陆风化 (Pogge von Strandmann等, 2013) 导致大气 $\mathrm{CO}_{2}$ 含量的 降低.

\section{3 两类极热事件启动阶段的增温幅度与速率}

这五次极热事件的增温几乎都与碳同位素的启动 阶段同步，因此本文利用碳同位素启动阶段持续的时 间及其对应的增温幅度来计算升温速率. 在实际计算 过程中优先采用来自各事件的金钉子剖面或典型钻孔 上的启动阶段的时间限定，而增温幅度则采用文献所 报道的数据, 以此获得的五次增温事件的增温速率 (图4).

\subsubsection{NCHE事件}

PTB期间的温度主要来自低纬度牙形石氧同位素 数据, 增温主要发生在启动阶段及负平台阶段底部, 增 温幅度约为 $8 \sim 10^{\circ} \mathrm{C}$, 若以煤山剖面启动持续时间 $\sim 60 \mathrm{kyr}$ (Burgess等, 2014)计算, 则增温速率约为 $0.133 \sim$ $0.167^{\circ} \mathrm{C} \mathrm{kyr}^{-1}$; TOAE期间的古温度数据主要来自中纬 度地区, 载体为腕足、双壳、箭石和鱼骨等, 事件启动 阶段增温幅度约为7 $10^{\circ} \mathrm{C}$, 若以葡萄牙Peniche剖面启 动阶段持续时间 150kyr 计算(Suan等, 2008b), 则 TOAE增温速率约为 $0.047 \sim 0.067^{\circ} \mathrm{C} \mathrm{kyr}^{-1}$; $\mathrm{PETM}$ 温度 数据主要来源于浮游有孔虫氧同位素和 $\mathrm{TEX}_{86}$, 增温 主要发生在事件启动阶段, 从低纬度到高纬度增温幅 度依次增大, 分别约为3 5、6 8、5 9 ${ }^{\circ} \mathrm{C}$, 而PETM的 启动阶段持续时间为 $3 \sim 5 \mathrm{kyr}$ (Zeebe等, 2009; Bowen等, 2015; Frieling等, 2017; Turner, 2018). 若以5kyr计算, PETM启动阶段在低、中和高纬度的最小增温速率分 别约为 $0.6 \sim 1.0 、 1.2 \sim 1.6 、 1.0 \sim 1.8^{\circ} \mathrm{C} \mathrm{kyr}^{-1}$.

\subsubsection{PCHE事件}

OAE1a的温度记录主要来自 $\mathrm{TEX}_{86}$, 增温主要发 生在事件启动阶段, 从低纬度到高纬度增温幅度依次 约为 5 6、2 4和 $2 \sim 3^{\circ} \mathrm{C}$, 若以土耳其Yenicesihlar剖面 上启动阶段的持续时间 $75 \mathrm{kyr}$ 计算 $(\mathrm{Hu}$ 等，2012), 则其 

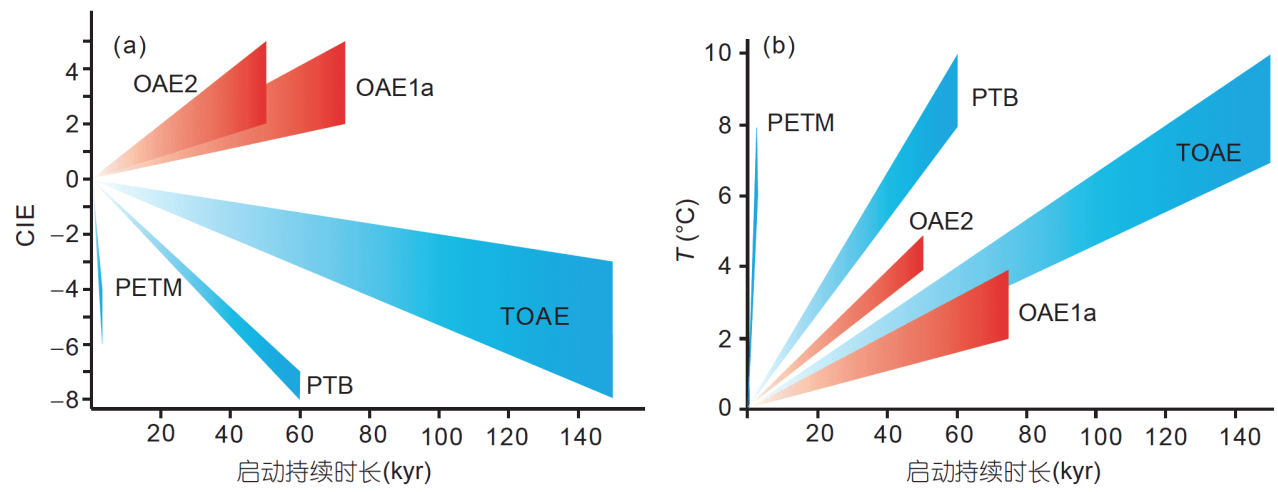

图 4 五次极热事件启动阶段碳同位素偏移幅度与偏移速率(a)、增温幅度与增温速率(b)的对比 除PTB以低纬度温度计算, 其他事件均以高纬度温度计算, 具体参考文献见表 1

低、中和高纬度的增温速率分别约为 $0.067 \sim 0.080$ 、 $0.027 \sim 0.053 、 0.027 \sim 0.040^{\circ} \mathrm{C} \mathrm{kyr}^{-1}$. OAE2 的温度记录 主要来自浮游有孔虫氧同位素和 $\mathrm{TEX}_{86}$, 增温主要发 生在事件启动阶段，从低纬度到高纬度增温幅度依次 增大，分别约为2 3、4 5和7 $10^{\circ} \mathrm{C}$ ，若采用藏南贡扎 剖面启动阶段持续时间 $50 \mathrm{kyr}$ 计算 $(\mathrm{Li} \mathrm{Y} \mathrm{X}$ 等，2017), OAE2在低、中和高纬度的增温速率约为 $0.04 \sim 0.06$ 、 $0.08 \sim 0.1$ 和 $0.14 \sim 0.2^{\circ} \mathrm{C} \mathrm{kyr}^{-1}$.

根据本文综述和计算, 这五次极热事件中, PTB事 件是这五次极热事件中增温幅度最大的, 随后依次为 TOAE、OAE1a、PETM、OAE2(图4); 而PETM的增 温速率最快, 大于 $1{ }^{\circ} \mathrm{C} \mathrm{kyr}^{-1}$, 其后依次是 $\mathrm{PTB}$ 、 OAE1a、OAE2、TOAE(图4). 相比较而言, 现今全球 气温增温速率 (取 150 年增温 $1^{\circ} \mathrm{C}$ ) 是PETM启动期间中 纬度地区增温速率的4.2 5.6倍.

\section{5 中生代-古近纪两类极热事件引起的环境 变化}

\section{1 两类极热事件引起的海相沉积变化}

五个极热事件在浅海环境都有沉积记录. 由于板 块运动等原因, 现今仅OAE1a、OAE2和PETM保存有 深海沉积记录.

\subsubsection{NCHE引起的海相沉积变化}

(1) 浅海环境碳酸盐台地死亡. 碳酸盐台地死亡是 指相对海平面上升速率超过浅海生态系统碳酸盐堆积 速率时, 碳酸盐台地或生物礁没入透光带以下, 或受到 陆源输入影响出现生物生产力降低甚至消亡的现象
(Godet，2013).PTB事件期间碳酸盐台地和生物礁死 亡, 伴随层状硅质岩和煤消失, 以及黑色页岩、泥质 白云岩、黄铁矿、火山灰、微生物岩等的出现(曹长 群和郑全锋, 2009); TOAE事件期间, 生物碳酸盐台地 死亡, Lithiotis灰岩被深灰色的斑点灰岩、微晶灰岩取 代(Sabatino等, 2013; Han等, 2016, 2018), 或者转变为 无生物的鲌粒灰岩(Trecalli等, 2012); PETM事件期间, 碳酸盐缓坡发生死亡，碳酸盐岩沉积被砾岩 $(\mathrm{Li} \mathrm{J}$ 等， 2017), 或含大量陆源碎屑的钙质泥灰岩沉积打断( $\mathrm{Pu}-$ jalte等, 2014).

(2) 出现特殊的沉积类型. PTB事件时期在大陆边 缘地区广泛出现“错时相”沉积, 包括扁平砾石砾岩、 蠕虫状灰岩、微生物岩、碳酸盐岩海底扇、薄层灰 岩、条带状灰岩、巨鲕灰岩等(赵小明等，2008; 李飞 等，2010). TOAE事件时期特提斯和泛大洋热带/亚热 带地区频繁出现具侵蚀面、渠模、丘状层理等沉积构 造的风暴沉积(Krencker等, 2015; Han等, 2018; Izumi 等, 2018). PETM事件时期在浅海碳酸盐台地环境下, 碳酸盐岩被砾岩层(Li J 等, 2017)或砂质、钲质泥灰岩 替代(Pujalte等, 2014), 而在深海环境下钻质泥灰岩被 黍土岩替代(Zachos等, 2005).

\subsubsection{PCHE引起的海相沉积变化}

(1) 浅海环境下碳酸盐台地部分死亡. OAE1a期间 北特提斯碳酸盐岩台地死亡, 出现凝缩段和硬底构造 (Skelton和Gili, 2012), 而南特提斯大陆边缘碳酸盐岩 台地继续生长, 出现富含Lithocodium-Bacinella或orbitolinid的沉积(Husinec等, 2012). OAE2期间, 西特提斯 西班牙北部比利牛斯山地区(Drzewiecki和Simo, 
1997)、克罗地亚西部地区(Korbar等，2012)以及土耳 其东南(Mülayim等, 2019), 出现浅水碳酸盐台地死亡.

(2) 深水环境出现富有机质的黑色页岩. OAE1a和 OAE2事件前后均为远洋灰岩, 而在事件启动阶段均转 变为黑色页岩或富含有机质沉积(van Bentum等, 2009; $\mathrm{Hu}$ 等, 2012; Patruno等, 2015; Li等, 2016).

\section{2 两类极热事件引起的古海洋环境变化}

本文讨论的极热事件引起的古海洋环境变化主要 包括氧化还原条件、海洋酸化和海平面变化三个 方面.

\subsubsection{NCHE引起的古海洋变化}

(1) 海洋出现不同程度的缺氧, 但是缺氧范围有待 进一步厘清. PTB期间出现富机质含量的黑色页岩、 草莓状黄铁矿、绿硫细菌繁盛等指示缺氧环境的指标 (Cao等, 2009). TOAE期间在欧洲北部出现强烈的缺氧 环境, 而在欧洲南部则出现不缺氧或贫氧环境(Jenkyns 等, 2010). PETM期间全球海洋含氧量降低(Harding等, 2011; Winguth等，2012)，深海环境下出现缺氧或低氧 条件(Pälike等，2014)，而浅海大陆架、斜坡环境下出 现季节性缺氧或贫氧(Speijer和Wagner, 2002; Sluijs等, 2014).

(2) 海洋出现酸化, 但是深水与浅水环境下的酸化 程度有待进一步查明. 关于PTB期间的海洋酸化, 目前 存在较大争议, 一部分学者根据碳酸盐岩钻同位素研 究认为在事件启动阶段出现海洋酸化(Payne等，2010; Hinojosa等，2012), 而另一些学者根据碳酸盐岩硼同 位素研究认为, 在事件负平台阶段发生海洋酸化 (Clarkson等, 2015). TOAE期间钻同位素负偏移，指示 海洋发生酸化(Brazier等，2015). PETM时期深海环境 下普遍出现 $\mathrm{CaCO}_{3}$ 溶解, 且随水深加深, $\mathrm{CaCO}_{3}$ 的溶解 程度增加(Zachos等, 2005), 同时浮游有孔虫硼同位素 组成 $\left(\delta^{11} \mathrm{~B}\right)$ 和 $\mathrm{B} / \mathrm{Ca}$ 比值降低, 指示海水表层和溶跃层 $\mathrm{pH}$ 值降低(Penman等, 2014).

(3) 海平面变化争议较大. PTB期间, Jiang等(2014) 和Yin等(2014)认为在事件启动阶段存在一次海退，以 煤山第24层顶部“铁壳层”或大贵州滩微生物岩底部的 溶蚀面为标志, 而曹长群和郑全锋(2009)则认为“铁壳 层”或溶蚀面是海洋酸化引起，而非海退的结果. TOAE期间，在事件启动阶段之前存在一次短暂的海
平面下降，随后海平面上升(Haq，2018；Krencker等， 2019). PETM事件与海平面变化之间有两种观点，一 种认为在PETM事件启动阶段之前20 200kyr，发生海 平面上升, 海岸线向陆地方向迁移(Speijer和Wagner, 2002; Harding等, 2011; Sluijs等, 2008); 另一种观点认 为在PETM事件启动阶段之前, 存在一次短暂的海平 面下降，随后海平面上升(Schmitz和Pujalte，2003， 2007; Pujalte等, 2014).

\subsubsection{PCHE引起的古海洋变化}

（1）氧化还原条件不一, 具时空异性. 例如, OAE1a 期间, 在西特提斯深水环境下广泛出现缺氧局限环境, 而在浅水或大陆边缘环境下则出现不缺氧或贫氧环境 (Westermann等, 2013). OAE2期间, 原北大西洋深水环 境普遍出现缺氧环境(Pancost等, 2004), 其中南部地区 缺氧更甚(van Bentum等, 2009, van Helmond等, 2014); 在北大西洋Demera Rise和摩洛哥地区陆架环境下, 出 现铁化与缺氧条件交替(van Bentum等, 2009); 但是在 中纬度原北大西洋北部, 在缺氧条件下, 亦见短暂的富 氧(van Helmond等, 2014; Goldberg等, 2016).

(2) 洋流可能发生重组. 鱼骨化石Nd同位素偏移 记录了古海洋洋流的改变, OAE2期间, 北大西洋 ODP1258钻孔中鱼骨化石 $N d$ 同位素研究发现 $\varepsilon_{\mathrm{Nd}}$ 正偏 移8个单位(MacLeod等, 2008), 而在英国Eastbourne剖 面上，鱼骨化石 $\varepsilon_{\mathrm{Nd}}$ 先负偏一个单位，后正偏 3 个单位 (Zheng等, 2013).

(3) 海洋酸化、海平面变化报道较少. 但是地质记 录表明, 即使两极无冰的白严纪Turonian期也存在快 速海平面变化事件(Haq和Huber, 2017).

\section{3 两类极热事件引起的陆相沉积环境变化}

\subsubsection{NCHE引起的陆相沉积环境变化}

(1) 陆相极端干旱环境频发. TOAE期间中国北方 地区广泛出现杂色或红色沉积物, 指示聚煤作用减弱, 喜热、耐干型植物的数量增加(邓胜徽等, 2012); 北美 Bighorn盆地古土壤证据表明，极端干旱环境出现在 PETM启动之前, 一直持续至PETM恢复期才转变为相 对潮湿型气候(Wing等, 2005; Kraus和Riggins, 2007).

（2）陆地水循环增强, 河流形态发生改变. PTB期 间, 出现大规模的河流类型的转变, 事件前后为曲流河 沉积体系, 而事件期间突然转变为辫状河沉积体系 
(Ward等, 2000; Shen等, 2011; Zhu等, 2019). PETM期 间，西班牙Tremp-Graus盆地的水流量增加至少 1.35 倍， 最高可达14倍(Chen等, 2018), 河漫滩泥质沉积被河流 冲积砾岩取代, 为巨大的洪水, 河流改道频繁, 河道宽 度大幅度拓宽的结果(Chen等, 2018). 在美国北Bighorn 盆地，PETM上下地层为薄层古土壤和厚层泛滥平原 沉积，而PETM期间为厚层古土壤层和薄层泛滥平原 沉积或厚层河道砂岩沉积(Kraus等, 2015).

\subsubsection{PCHE引起的陆相沉积环境变化}

目前关于PCHE对陆相沉积环境影响的研究甚少, 主要原因在于陆相沉积记录较少. PCHE期间营养物质 输入增加, 初级生产力增强, 湖泊季节性或永久缺氧, 发育富含有机质的黑色泥岩(Jenkyns, 2010). 干旱潮湿 条件不一, 具时空异性. 例如, OAE1a期间, 昌马盆地为 较潮湿的气候和季节性干燥气候(Suarez等, 2017); 而 北美犹他州前陆盆地则为干旱气候(Ludvigson等, 2010).

\section{6 中生代-古近纪两类极热事件引起的生物 变化}

定量数据研究显示, NCHE类PTB、TOAE、 PETM事件和PCHE类OAE1a、OAE2事件，其科级生 物绝灭率分别为 $55.7 \% 、 17.3 \% 、 7.6 \%$ 和 $9.9 \% 、 13.6 \%$ (Sepkoski，1996)，其中PTB、TOAE、OAE2三次事件 被列入显生宙18次生物大绝灭事件(Bambach，2006). 由此可见，相比较而言，NCHE类极热事件比PCHE类 极热事件对生物的影响更为显著，而PETM生物绝灭 率低的原因很可能是其高温持续时间较短.

\subsection{NCHE与生物变化}

\subsection{1 海洋生物变化}

(1) 海洋生物出现低纬度向高纬度迁移现象. TOAE和PTB事件期间，菊石、有孔虫、介形虫、箭 石等受到影响，且向极地迁移(Dera等，2011; Bernardi 等, 2018). PETM期, 特提斯低、中纬度以珊瑚为主的 浅海碳酸盐台地，逐步变成以底栖大有孔虫为主要生 物的浅水碳酸盐台地, 由于温度升高, 珊瑚从低纬度 向中纬度地区迁移, 直至在低中纬度地区消失(Scheibner和Speijer, 2008).
(2) 海洋生物发生不同程度的绝灭或更替. 一些对 温度、含氧量、 $\mathrm{pH}$ 等海洋条件敏感的生物, 发生不同 程度的绝灭或更替. PTB期间, 铂藻、䗴、四射珊瑚、 横板珊瑚、三叶虫、放射虫等栖息环境较窄的生物发 生彻底灭绝(沈树忠和张华, 2017), 而一些栖息环境较 广的小有孔虫、介形虫、腕足动物、软体动物、菊 石、牙形石等灭绝率相对较低(Song等, 2013). TOAE 时期特提斯南缘低纬度地区浅水碳酸盐台地上Lithiotis双壳发生绝灭(Trecalli等，2012; Sabatino等，2013). PETM时期深海环境底栖小有孔属种绝灭率为 $30 \%$ (Takeda和Kaiho, 2007), 钙质超微化石生物则出现18属 更替或灭绝(Gibbs等, 2006), 而在浅水碳酸盐台地上晚 古新世底栖大有孔虫组合Ranikothalia-Miscellanea被 始新世底栖大有孔虫组合Nummulites-Alveolina替代 (Scheibner等, 2005).

(3) 海洋生物丰度、形态等发生变化. PTB期间, 浅海生物如底栖有孔虫、双壳、腹足、腕足、介形 虫、牙形石、鱼以及陆相介形虫和四足动物等体型变 小, 而蓝细菌由于缺少竞争和天敌, 在事件之后快速占 据主导地位形成微生物岩(Wilson和MacArthur, 1967). TOAE期间，西特提斯的化石资料表明海相软体动物 和小型浮游生物的个体发生明显减小 (Morten 和 Twitchett, 2009). PETM期间, 一些沟鞭藻类异常繁盛, 丰度和分异度增大(Sluijs和Brinkhuis, 2009); 底栖小有 孔虫体型急剧变小, 而浮游有孔虫体型变大(Kaiho等, 2006).

\subsection{2 陆地生物变化}

（1）陆地生物从低纬度向高纬度迁移. TOAE期 间, 喜热、耐干型分子的比例显著增加且向高纬度迁 移(邓胜徽等，2012); PETM期间北美Bighorn盆地松 柏类和阔叶林植物群落被豆类植物所替代, 植物群落 向北迁移了650 1500km(Wing等, 2005), 此外一些哺 乳动物和蚚蜴类也发生了迁移(McInerney和Wing, 2011).

（2）陆地生物发生不同程度的绝灭或爆发. PTB期 间，华南地区以大羽羊齿为代表的热带雨林植物群基 本消失(Shen等, 2011; Yu等, 2015; Bernardi等, 2018), 二叠纪晚期占主导地位的Dicynodon动物群被以 $L y$ strosaurus 为特征的动物群替代(Ward等, 2005; Gastaldo 等, 2019). PETM期间鳄龙属、哺乳动物中近猴科发 
生绝灭，但奇蹄类、偶蹄类、啮齿类及灵长类等新物 种呈“爆发式”突然出现(Gingerich, 2003).

\subsection{PCHE与生物变化}

PCHE事件对陆地生物和浅海生物没有造成明显 的影响, 但是一些对环境变化比较敏感的深水生物种 属发生部分绝灭或增生。例如，OAE1a期间钻质超微 生物出现明显的危机(Erba等，2010)。 OAE2期间浮游 轮孔虫属(Rotalipora) 发生绝灭, 而浮游异卷虫属 (Heterohelix)发生明显增生(Lecki等，2002；Erba，2004). 此外，OAE1a、OAE2期间放射虫属也发生明显的绝 灭, 绝灭率分别为 $41 \%$ 和 $58 \%$ (Erbacher等, 1996).

\section{7 中生代-古近纪两类极热事件的驱动机制}

\subsection{NCHE的驱动机制}

NCHE类极热事件表现为大量轻碳注入到大气-海 洋系统, 导致碳同位素负偏移. 我们认为, 这类极热事 件的驱动机制可能是陆地环境(包括浅海环境)喷发的 大火成岩省(本文称之为大陆火成岩省, LIP; 图 5a).

(1) NCHE类极热事件证实与大陆大火成岩省相 关. 高精度的定年技术限定这类极热事件与大火成岩 省喷发在时间上的一致性，如PTB与Siberian LIP、 TOAE与Karoo-Ferrar LIP、PTEM与北大西洋LIP, 且 这几次LIPs都毫无例外地发生在大陆环境(Wignall, 2001; McElwain等, 2005; Bond和Wignall, 2014; Burgess等, 2015, 2017; Ernst和Youbi, 2017).

(2) 虽然关于碳的来源一直存在较大争议, 主要存 在大火成岩省喷发和天然气水合物释放两种不同观 点, 本文赞成前者. 大火成岩省喷发或者浅层侵入到碳 酸盐岩(灰岩、白云岩)、硫酸盐岩(蒸发岩)、有机岩 (煤炭、黑色页岩等), 导致大规模 $\mathrm{CO}_{2} 、 \mathrm{CH}_{4} 、 \mathrm{H}_{2} \mathrm{~S}$ 等 气体的排放(Gutjahr等, 2017), 使得巨量轻碳进入到大 气-海洋系统, 从而导致碳同位素负偏移和全球增温.

(3) 大陆大火成岩省喷发造成大气-海洋系统的温 度、环境、生物发生明显变化. 由于陆地或浅海喷发, 最先影响的是大气陆地环境, 导致全球变暖、野火和 干旱频繁、酸雨、大陆风化增强、陆源输入增加、臭 氧层破坏等(Ernst和Youbi, 2017). 海洋环境下, 碳酸盐 台地消亡、海洋酸化、大洋缺氧等出现, 海相生物发 生绝灭、更替或迁移(戎嘉余和黄冰，2014; 宋海军和
童金南，2016；Bond和Grasby，2017；沈树忠和张华， 2017; Benton, 2018).

\subsection{PCHE的驱动机制}

PCHE类极热事件主要表现为仅在启动时碳同位 素发生短暂的负偏移，随后很快发生大规模有机质埋 藏, 导致碳同位素正偏移. 本文认为, 此类事件的驱动 机制可能是深海环境喷发的大火成岩省(本文称之为 大洋大火成岩省; 图 $5 b$ ).

(1) PCHE类极热事件证实与大洋大火成岩省相 关. 高精度的定年技术限定这类极热事件与大洋大火 成岩省喷发在时间上的一致性, Ontong Java LIP与 OAE1a、Caribean LIP与OAE2以及Kergenlian LIP与 白垩纪其他几次大洋缺氧事件 $(O A E 1 b 、 O A E 1 c$ 、 OAE1d)在时间上都存在很好的对应关系(Wignall, 2001; Ernst和Youbi, 2017).

(2) PCHE类极热事件为大洋大火成岩省喷发触 发. 大洋火成岩省喷发初期, 巨量轻碳进入到大气-海 洋系统中, 引起碳同位素短暂负偏移和全球增温, 但是 由于其在深海环境喷发, 释放的热量和营养物质, 直接 进入海洋系统, 一方面由于喷发高温使得海水溶解氧 含量减少，造成海水缺氧; 另一方面大陆风化及岩浆 喷发释放使得海水表层营养物质的增加, 海洋初始生 产力增加, 造成海底缺氧. 海水缺氧, 导致有机质大规 模埋藏, 碳无法返回到大气-海洋系统, 碳同位素发生 正偏移.

(3) 大洋大火成岩省喷发造成全球深海普遍缺 氧、有机质大量埋藏和黑色页岩沉积, 浅水碳酸盐岩 台地部分死亡，对陆地、浅海生物并未造成明显的变 化, 对深海环境的生物造成较小影响, 如出现钙质超 微危机等(Erba等, 2010).

\section{8 潜在应用: 地质历史时期重大气候事件驱 动机制的判别}

根据现有资料, 本文认为三叠纪-侏罗纪界线事件 ( 201Ma)(对应于Camp大火成岩省)、白严纪-古近纪 界线事件( 66 Ma) (对应于Deccan大火成岩省)均属于 $\mathrm{NCHE}$ 类极热事件, 而其驱动机制为大陆大火成岩省; 早白严世Valangian OAE事件( 132Ma)(对应于ParanaEtendeka、Bunbury-Comei大火成岩省)(网络版附表 

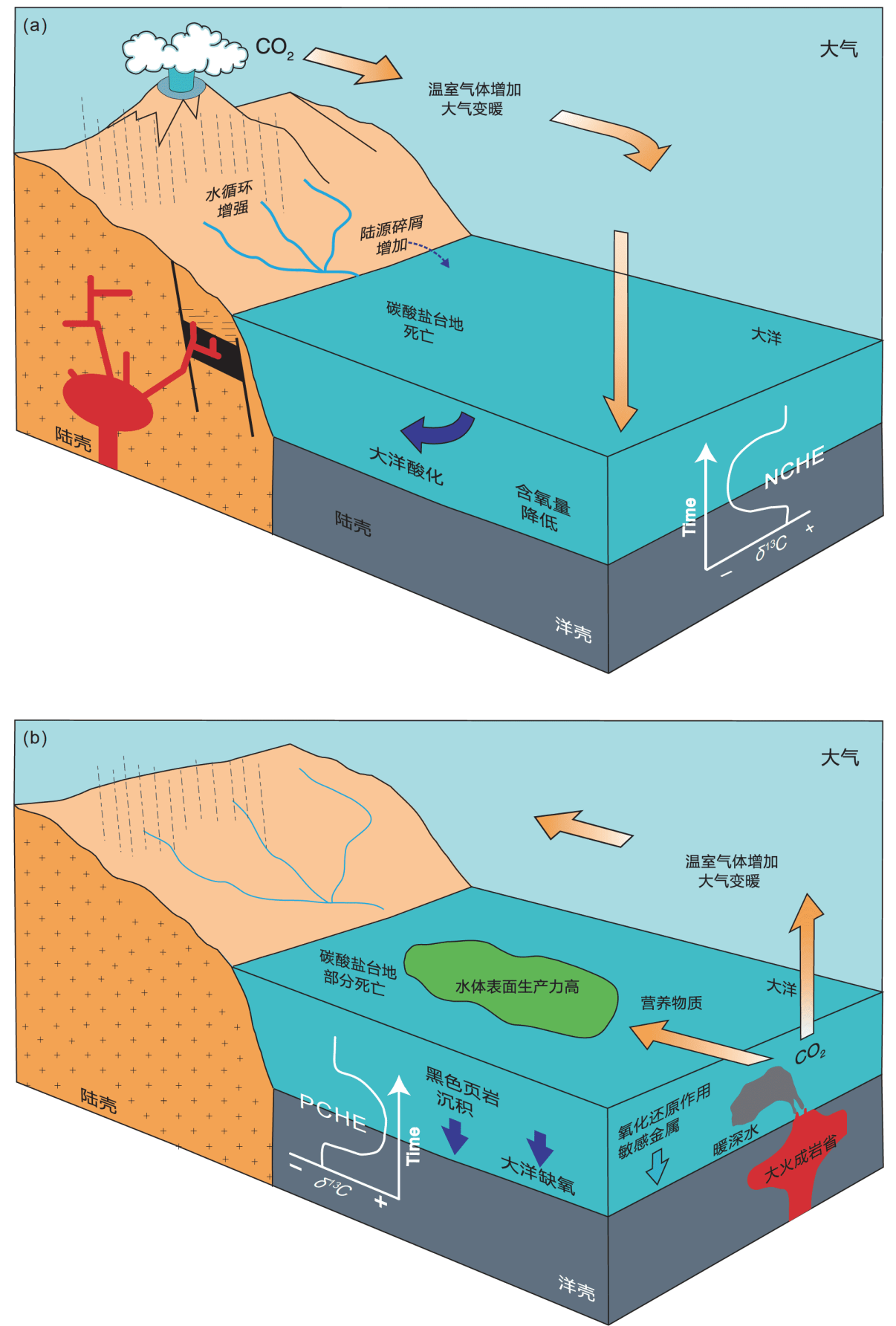

图 5 NCHE与PCHE两类极热事件的驱动机制及其引起的气候、环境变化示意图

(a) NCHE类极热事件的驱动机制是陆地环境喷发的大火成岩省; (b) PCHE类极热事件的驱动机制是深海环境喷发的大火成岩省. 修改自 Robinson等(2017)

$\mathrm{S} 1, \mathrm{http}: / /$ earthen.scichina.com), 为PCHE类的极热事件, 而其驱动机制为大洋大火成岩省(附表S1)。这些事件 无一例外都保存有良好的岩浆岩记录与沉积地层，因
此可以建立起成因上的联系．限于篇幅，本文不再对 这些事件进行论述，感兴趣的读者可进一步查阅相关 论文. 但是，众所周知，由于板块俯冲，晚侏罗世之前 
大洋环境下形成的大火成岩省，多被俯冲消减掉或以 零散的方式保留在造山带中，而此期间发生的重大地 质事件，常因找不到对应时代的岩浆岩记录而无法建 立起成因上的联系. 因此，本文认为在确认属于极热 事件的前提下，可以根据其沉积、古海洋、碳同位 素、古生物等特征, 确定其为哪类极热事件, 据此推 断其驱动机制.

实例 1. 晚三叠世 C a r n i a n 期洪积事件 (约 234 232Ma, CPE): 全球海水表层温度升高约 $6 \sim 8^{\circ} \mathrm{C}$, 碳同位素发生-4.2\%o偏移(Dal Corso等, 2012; Sun等, 2019). 事件期间气候转变为湿润、降雨量增多、陆源 硅质碎屑输入增强, 碳酸盐岩沉积被打断并转变为陆 源碎屑岩沉积(Simms和Ruffell, 1989; 金金等，2015). 陆地植物、浅海无脊椎动物均发生不同程度的更替或 绝灭, 如高灭绝率的菊石、海百合, 钙质浮游生物大规 模爆发; 陆地上恐龙首次出现，针叶类植物高度繁盛. 根据该事件表现的碳同位素、环境和生物特征, 认为 其为NCHE类极热事件，因此其驱动机制可能为大陆 大火成岩省喷发. 众多学者认为Carnian期洪积事件驱 动机制为北美Wrangellia大火成岩省的喷发，而Wrangellia玄武岩主要分布在加拿大西海岸至阿拉斯加南 部地区, 其下部为中晚Ladinian期含Daonella黑色页岩, 上部为Carnian晚期-Norian中期浅水碳酸盐地层(Stanley, 1989), 限定其喷发环境为大陆边缘浅海, 为大陆喷 发环境的大火成岩省，符合大陆大火成岩省喷发驱动 NCHE事件的推论.

实例2. 早-中寒武世界线事件( $510 \mathrm{Ma})$ : 该界线事 件出现碳同位素负偏、硫同位素正偏、海平面上升 (Montañez等, 2000; Hough等, 2006) 以及广泛的大洋缺 氧(Zhuravlev和Wood, 1996)和大量生物灭绝(Sepkoski, 1996). 根据该事件表现的生物和环境响应，判断其为 NCHE类极热事件，因此其驱动机制可能为大陆大火 成岩省喷发. 近年来, 澳大利亚西部Kalkarindji大火成 岩省的时代被精确限定为(510.7 \pm 0.6$) \mathrm{Ma}$ (Jourdan等, 2014), 与早-中寒武世界线事件在时间上高度一致, 且 该大火成岩省喷发于陆相环境(Jourdan等, 2014), 符合 大陆大火成岩省喷发驱动NCHE事件的推论.

实例3. Frasnian-Famennian事件( 372Ma): 事件时 期海水表层增温 $9^{\circ} \mathrm{C}($ Joachimski等, 2009), 碳同位素 正偏移超过 3.0\%o(Joachimski等, 2002; Xu等, 2012), 海 洋广泛出现缺氧条件和黑色页岩沉积(Murphy等,
2000). 该事件是显生宙五大集群灭绝事件之一，但是 受影响的主要是低纬度地区和浅水生态系统, 而高纬 度地区和陆地生态系统所受影响较小. 根据该事件表 现的碳同位素、沉积环境和生物特征, 判断其为 PCHE类极热事件, 其驱动机制可能为大洋大火成岩 省喷发. Frasnian-Famennian事件附近出现多期次 Hg富 集，指示了火山事件的作用(Racki等, 2018), 但是未见 存在大规模火山岩的直接证据, 因此, 其驱动机制有待 今后研究的进一步证实.

\section{9 结论与展望}

本文从中生代-古近纪五次极热事件研究出发, 通 过对这些事件的碳同位素、持续时间、增温幅度和速 率、沉积记录、古海洋条件、古生物等系统总结和深 入分析, 获得了一些有趣的定量数据, 为认识当前人类 面临的全球气候变暖和判断地质历史时期重大气候事 件驱动机制提供了重要的参考和启示. 主要结论如下:

(1) 根据碳同位素特征, 把极热事件分为两类: NCHE类极热事件以碳同位素总体负偏移为特征，包 括PTB、TOAE、PETM，其驱动机制是大陆环境喷发 的大火成岩省; PCHE类极热事件以碳同位素总体正 偏移为特征, 包括OAE1a、OAE2, 其驱动机制是深海 环境喷发的大火成岩省.

(2) NCHE类极热事件表现为大量轻碳释放到大 气-海洋系统, 导致了碳同位素负偏移. 大陆环境下形 成大火成岩省，造成全球增温，极端干旱和野火频发， 大陆风化增强和陆源碎屑输入增多, 浅海碳酸盐台地 消亡, 大洋出现酸化、缺氧、生物大量灭绝等.

(3) PCHE类极热事件表现为大规模有机质埋藏, 导致了碳同位素正偏移。深海环境喷发的大火成岩省 主要对深海环境和生物产生一定影响, 对全球大气增 温和浅海和陆地环境、生物的影响不甚明显，造成大 洋广泛缺氧, 黑色页岩沉积, 部分碳酸盐岩台地死亡.

认识过去是为了更好地预测未来. 深时极热事件 为认识人类当前面临的全球气候变暖提供了独一无二 的可供对比和借鉴的研究实例. 2017年, SCIENCE CHINA Earth Sciences曾出版一个专题(Hu等，2017), 聚焦白严纪温室气候与海平面变化. 2018年, 英国皇家 学会以“极热事件: 在地质过去发生的快速而极端的全 球变暖”为主题组织了学术讨论会, 并出版了同名专辑 
的11篇论文(Foster等，2018)。这些研究成果集中反映 了对温室气候及其发生的极热事件的认识水平。限于 地质记录保留的不完整性和气候-环境参数的不精确 性, 科学界对极热事件的认识还存在局限性和片面性, 这有赖于更多高精度、可靠的定量数据的获取. 加强 深时古气候研究(王成善等, 2017), 尤其是对极热事件 的研究,无论对于了解地球的过去还是认识地球和人 类的未来都有极其重要的理论和现实意义.

致谢感谢王成善院士、郭正堂院士、沈树忠院士、 David Kemp教授、Micha Ruhl博士、崔莹博士、黄春菊 教授、陈吉涛研究员等富有建设性的讨论和意见. 感谢 六名若名评审人和胡建芳研究员的意见与建议，使得本 文质量有了明显提升.

\section{参考文献}

曹长群, 郑全锋. 2009. 煤山二叠纪-三叠纪过渡期事件地层时序的微

观地层记录. 中国科学: 地球科学, 39: 481-487

邓胜徽, 卢远征, 茹焚, 方林浩, 李金, 刘璐. 2012. 早侏罗世Toarcian 期大洋缺氧事件及其在陆地生态系统中的响应. 中国地质大学 学报, 37: 23-38

金鍂, 时志强, 王艳艳, 段雄, 程明. 2015. 晚三叠世中卡尼期极端气 候事件: 研究进展及存在问题. 沉积学报, 33: 105-115

李飞, 王夏, 薛武强, 颜佳新. 2010. 一种新的错时相沉积物——巨鲕 及其环境意义. 沉积学报, 28: 585-595

戎嘉余, 黄冰. 2014. 生物大灭绝研究三十年. 中国科学: 地球科学, 44: $377-404$

沈树忠, 张华. 2017. 什么引起五次生物大灭绝? 科学通报, 62: 11191135

宋海军, 童金南. 2016. 二叠纪-三叠纪之交生物大灭绝与残存. 地球 科学, 41: 901-918

王成善, 王天天, 陈㬢, 高远, 张来明. 2017. 深时古气候对未来气候 变化的启示. 地学前缘, 24: 1-17

赵小明, 童金南, 姚华舟, 张克信, Chen Z. 2008. 华南早三叠世错时 相沉积及其对复苏期生态系的启示. 中国科学: 地球科学，38: 1564-1574

Aze T, Pearson P N, Dickson A J, Badger M P S, Bown P R, Pancost R D, Gibbs S J, Huber B T, Leng M J, Coe A L, Cohen A S, Foster G L. 2014. Extreme warming of tropical waters during the PaleoceneEocene thermal Maximum. Geology, 42: 739-742

Aziz H A, Hilgen F J, van Luijk G M, Sluijs A, Kraus M J, Pares J M, Gingerich P D. 2008. Astronomical climate control on paleosol stacking patterns in the upper Paleocene-lower Eocene Willwood Formation, Bighorn Basin, Wyoming. Geology, 36: 531-534
Bains S, Corfield R M, Norris R D. 1999. Mechanisms of climate warming at the end of the Paleocene. Science, 285: 724-727

Bambach R K. 2006. Phanerozoic biodiversity Mass extinctions. Annu Rev Earth Planet Sci, 34: 127-155

Benton M J. 2018. Hyperthermal-driven Mass extinctions: Killing models during the Permian-Triassic Mass extinction. Phil Trans R Soc A, 376: 20170076

Bernardi M, Petti F M, Benton M J. 2018. Tetrapod distribution and temperature rise during the Permian-Triassic Mass extinction. Proc R Soc B, 285: 20172331

Bond D P G, Grasby S E. 2017. On the causes of Mass extinctions. Palaeogeogr Palaeoclimatol Palaeoecol, 478: 3-29

Bond D P, Wignall P B. 2014. Large igneous provinces and Mass extinctions: An update. In: Keller G, Kerr A C, eds. Volcanism, Impacts and Mass Extinctions: Causes and Effects. Spec Pap Geol Soc Am, 505: 29-55

Bottini C, Cohen A S, Erba E, Jenkyns H C, Coe A L. 2012. Osmiumisotope evidence for volcanism, weathering, and ocean mixing during the early Aptian OAE 1a. Geology, 40: 583-586

Boulila S, Galbrun B, Huret E, Hinnov L A, Rouget I, Gardin S, Bartolini A. 2014. Astronomical calibration of the Toarcian Stage: Implications for sequence stratigraphy and duration of the early Toarcian OAE. Earth Planet Sci Lett, 386: 98-111

Bowen G J, Maibauer B J, Kraus M J, Röhl U, Westerhold T, Steimke A, Gingerich P D, Wing S L, Clyde W C. 2015. TwoMassive, rapid releases of carbon during the onset of the Palaeocene-Eocene thermal Maximum. Nat Geosci, 8: 44-47

Brand U, Jiang G, Azmy K, Bishop J, Montañez I P. 2012. Diagenetic evaluation of a Pennsylvanian carbonate succession (Bird Spring Formation, Arrow Canyon, Nevada, U.S.A.)—1: Brachiopod and whole rock comparison. Chem Geol, 308: 26-39

Brazier J M, Suan G, Tacail T, Simon L, Martin J E, Mattioli E, Balter V. 2015. Calcium isotope evidence for dramatic increase of continental weathering during the Toarcian oceanic anoxic event (Early Jurassic). Earth Planet Sci Lett, 411: 164-176

Burgess S D, Bowring S A, Fleming T H, Elliot D H. 2015. Highprecision geochronology links the Ferrar large igneous province with early-Jurassic ocean anoxia and biotic crisis. Earth Planet Sci Lett, 415: 90-99

Burgess S D, Bowring S, Shen S. 2014. High-precision timeline for Earth's most severe extinction. Proc Natl Acad Sci USA, 111: 33163321

Burgess S D, Muirhead J D, Bowring S A. 2017. Initial pulse of Siberian Traps sills as the trigger of the end-Permian Mass extinction. Nat Commun, 8: 164

Cao C, Love G D, Hays L E, Wang W, Shen S, Summons R E. 2009. 
Biogeochemical evidence for euxinic oceans and ecological disturbance presaging the end-Permian Mass extinction event. Earth Planet Sci Lett, 281: 188-201

Charles A J, Condon D J, Harding I C, Pälike H, Marshall J E A, Cui Y, Kump L, Croudace I W. 2011. Constraints on the numerical age of the Paleocene-Eocene boundary. Geochem Geophys Geosyst, 12: Q0AA17

Chen C, Guerit L, Foreman B Z, Hassenruck-Gudipati H J, Adatte T, Honegger L, Perret M, Sluijs A, Castelltort S. 2018. Estimating regional flood discharge during Palaeocene-Eocene global warming. Sci Rep, 8: 13391

Chen J, Shen S, Li X, Xu Y, Joachimski M M, Bowring S A, Erwin D H, Yuan D, Chen B, Zhang H, Wang Y, Cao C, Zheng Q, Mu L. 2016. High-resolution SIMS oxygen isotope analysis on conodont apatite from South China and implications for the end-Permian Mass extinction. Palaeogeogr Palaeoclimatol Palaeoecol, 448: 2638

Clarkson M O, Kasemann S A, Wood R A, Lenton T M, Daines S J, Richoz S, Ohnemueller F, Meixner A, Poulton S W, Tipper E T. 2015. Ocean acidification and the Permo-Triassic Mass extinction. Science, 348: 229-232

Cui Y, Kump L R. 2015. Global warming and the end-Permian extinction event: Proxy and modeling perspectives. Earth-Sci Rev, 149: 5-22

Dal Corso J, Mietto P, Newton R J, Pancost R D, Preto N, Roghi G, Wignall P B. 2012. Discovery of a Major negative $\delta^{13} \mathrm{C}$ spike in the Carnian (Late Triassic) linked to the eruption of Wrangellia flood basalts. Geology, 40: 79-82

Dera G, Neige P, Dommergues J L, Brayard A. 2011. Ammonite paleobiogeography during the Pliensbachian-Toarcian crisis (Early Jurassic) reflecting paleoclimate, eustasy, and extinctions. Glob Planet Change, 78: 92-105

Dera G, Pucéat E, Pellenard P, Neige P, Delsate D, Joachimski M M, Reisberg L, Martinez M. 2009. Water Mass exchange and variations in seawater temperature in the NW Tethys during the Early Jurassic: Evidence from neodymium and oxygen isotopes of fish teeth and belemnites. Earth Planet Sci Lett, 286: 198-207

Drzewiecki P A, Simo J A. 1997. Carbonate platform drowning and oceanic anoxic events on a Mid-Cretaceous carbonate platform, south-central Pyrenees, Spain. J Sediment Res, 67: 698-714

Dumitrescu M, Brassell S C. 2006. Compositional and isotopic characteristics of organic Matter for the early Aptian oceanic anoxic event at Shatsky Rise, ODP leg 198. Palaeogeogr Palaeoclimatol Palaeoecol, 235: 168-191

Erba E. 2004. Calcareous nannofossils and Mesozoic oceanic anoxic events. Mar Micropaleontol, 52: 85-106
Erba E, Bottini C, Weissert H J, Keller C E. 2010. Calcareous nannoplankton response to surface-water acidification around Oceanic Anoxic Event 1a. Science, 329: 428-432

Erbacher J, Thurow J, Littke R. 1996. Evolution patterns of radiolaria and organic Matter variations: A new approach to identify sea-level changes in mid-Cretaceous pelagic environments. Geology, 24: 499-502

Ernst R E, Youbi N. 2017. How Large Igneous Provinces affect global climate, sometimes cause Mass extinctions, and represent natural Markers in the geological record. Palaeogeogr Palaeoclimatol Palaeoecol, 478: 30-52

Farley K A, Eltgroth S F. 2003. An alternative age model for the Paleocene-Eocene thermal Maximum using extraterrestrial ${ }^{3} \mathrm{He}$. Earth Planet Sci Lett, 208: 135-148

Forster A, Schouten S, Moriya K, Wilson P A, Sinninghe Damsté J S. 2007. Tropical warming and intermittent cooling during the Cenomanian/Turonian oceanic anoxic event 2: Sea surface temperature records from the equatorial Atlantic. Paleoceanography, 22: PA1219

Foster G L, Hull P, Lunt D J, Zachos J C. 2018. Placing our current 'hyperthermal' in the context of rapid climate change in our geological past. Phil Trans R Soc A, 376: 20170086

Frieling J, Gebhardt H, Huber M, Adekeye O A, Akande S O, Reichart G J, Middelburg J J, Schouten S, Sluijs A. 2017. Extreme warmth and heat-stressed plankton in the tropics during the PaleoceneEocene Thermal Maximum. Sci Adv, 3: e1600891

Gastaldo R A, Neveling J, Geissman J W, Li J. 2019. A multidisciplinary approach to review the vertical and lateral facies relationships of the purported vertebrate-defined terrestrial PermianTriassic boundary interval at Bethulie, Karoo Basin, South Africa. Earth-Sci Rev, 189: 220-243

Gibbs S J, Bown P R, Sessa J A, Bralower T J, Wilson P A. 2006. Nannoplankton extinction and origination across the PaleoceneEocene thermal Maximum. Science, 314: 1770-1773

Gingerich P D. 2003. Mammalian responses to climate change at the Paleocene-Eocene boundary: Polecat Bench record in the northern Bighorn Basin, Wyoming. Special Papers, 369: 463-478

Godet A. 2013. Drowning unconformities: Palaeoenvironmental significance and involvement of global processes. Sediment Geol, 293: 45-66

Goldberg T, Poulton S W, Wagner T, Kolonic S F, Rehkämper M. 2016. Molybdenum drawdown during Cretaceous oceanic anoxic event 2. Earth Planet Sci Lett, 440: 81-91

Gustafsson M, Holbourn A, Kuhnt W. 2003. Changes in Northeast Atlantic temperature and carbon flux during the Cenomanian/ Turonian paleoceanographic event: The Goban Spur stable isotope 
record. Palaeogeogr Palaeoclimatol Palaeoecol, 201: 51-66

Gutjahr M, Ridgwell A, Sexton P F, Anagnostou E, Pearson P N, Pälike

H, Norris R D, Thomas E, Foster G L. 2017. Very large release of mostly volcanic carbon during the Palaeocene-Eocene Thermal Maximum. Nature, 548: 573-577

Han Z, Hu X M, Li J, Garzanti E. 2016. Jurassic carbonate microfacies and relative sea-level changes in the Tethys Himalaya (southern Tibet). Palaeogeogr Palaeoclimatol Palaeoecol, 456: 1-20

Han Z, Hu X, Kemp D B, Li J. 2018. Carbonate-platform response to the Toarcian Oceanic Anoxic Event in the southern hemisphere: Implications for climatic change and biotic platform demise. Earth Planet Sci Lett, 489: 59-71

Haq B. 2018. Triassic eustatic variations reexamined. GSA Today, 28: 4-9

Haq B U, Huber B T. 2017. Anatomy of a eustatic event during the Turonian (Late Cretaceous) hot greenhouse climate. Sci China Earth Sci, 60: 20-29

Harding I C, Charles A J, Marshall J E A, Pälike H, Roberts A P, Wilson P A, Jarvis E, Thorne R, Morris E, Moremon R, Pearce R B, Akbari S. 2011. Sea-level and salinity fluctuations during the Paleocene-Eocene thermal Maximum in Arctic Spitsbergen. Earth Planet Sci Lett, 303: 97-107

Hesselbo S P, Gröcke D R, Jenkyns H C, Bjerrum C J, Farrimond P, Morgans Bell H S, Green O R. 2000. Massive dissociation of gas hydrate during a Jurassic oceanic anoxic event. Nature, 406: 392 395

Hesselbo S P, Jenkyns H C, Duarte L V, Oliveira L C V. 2007. Carbonisotope record of the Early Jurassic (Toarcian) Oceanic Anoxic Event from fossil wood and Marine carbonate (Lusitanian Basin, Portugal). Earth Planet Sci Lett, 253: 455-470

Hinojosa J L, Brown S T, Chen J, DePaolo D J, Paytan A, Shen S, Payne J L. 2012. Evidence for end-Permian ocean acidification from calcium isotopes in biogenic apatite. Geology, 40: 743-746

Hough M L, Shields G A, Evins L Z, Strauss H, Henderson R A, Mackenzie S. 2006. A Major sulphur isotope event at c $510 \mathrm{Ma}$ : A possible anoxia-extinction-volcanism connection during the EarlyMiddle Cambrian transition? Terra Nova, 18: 257-263

Hu X M, Wagreich M, Yilmaz I O. 2012. Marine rapid environmental/ climatic change in the Cretaceous greenhouse world. Cretac Res, 38: $1-6$

Hu X M, Wagreich M, Sames B. 2017. Special Topic: Cretaceous greenhouse palaeoclimate and sea-level changes. Sci China Earth Sci, 60: 1-4

Huang C J, Hesselbo S P. 2014. Pacing of the Toarcian Oceanic Anoxic Event (Early Jurassic) from astronomical correlation of Marine sections. Gondwana Res, 25: 1348-1356
Huber B T, MacLeod K G, Watkins D K, Coffin M F. 2018. The rise and fall of the Cretaceous Hot Greenhouse climate. Glob Planet Change, 167: 1-23

Husinec A, Harman C A, Regan S P, Mosher D A, Sweeney R J, Read J F. 2012. Sequence development influenced by intermittent cooling events in the Cretaceous Aptian greenhouse, Adriatic platform, Croatia. AAPG Bull, 96: 2215-2244

Intergovernmental Panel on Climate Change (IPCC). 2018. Special report of global warming of $1.5^{\circ} \mathrm{C}$. https://www.ipcc.ch/sr15/

Izumi K, Kemp D B, Itamiya S, Inui M. 2018. Sedimentary evidence for enhanced hydrological cycling in response to rapid carbon release during the early Toarcian oceanic anoxic event. Earth Planet Sci Lett, 481: 162-170

Jarvis I, Gale A S, Jenkyns H C, Pearce M A. 2006. Secular variation in Late Cretaceous carbon isotopes: $\mathrm{A}$ new $\delta^{13} \mathrm{C}$ carbonate reference curve for the Cenomanian-Campanian (99.6-70.6 Ma). Geol Mag, 143: 561-608

Jenkyns H C, Dickson A J, Ruhl M, Van Den Boorn S H J M. 2017. Basalt-seawater interaction, the Plenus Cold Event, enhanced weathering and geochemical change: Deconstructing Oceanic Anoxic Event 2 (Cenomanian-Turonian, Late Cretaceous). Sedimentology, 64: 16-43

Jenkyns H C, Forster A, Schouten S, Sinninghe Damsté J S. 2004. High temperatures in the Late Cretaceous Arctic Ocean. Nature, 432: 888-892

Jenkyns H C, Schouten-Huibers L, Schouten S, Sinninghe Damsté J S. 2012. Warm Middle Jurassic-Early Cretaceous high-latitude seasurface temperatures from the Southern Ocean. Clim Past, 8: 215226

Jenkyns H C. 2010. Geochemistry of oceanic anoxic events. Geochem Geophys Geosyst, 11: 1-30

Jiang H, Lai X, Sun Y, Wignall P B, Liu J, Yan C. 2014. PermianTriassic conodonts from Dajiang (Guizhou, South China) and their implication for the age of microbialite deposition in the aftermath of the End-Permian Mass extinction. J Earth Sci, 25: 413-430

Joachimski M M, Lai X, Shen S, Jiang H, Luo G, Chen B, Chen J, Sun Y. 2012. Climate warming in the latest Permian and the PermianTriassic Mass extinction. Geology, 40: 195-198

Joachimski M M, Breisig S, Buggisch W, Talent J A, Mawson R, Gereke M, Morrow J R, Day J, Weddige K. 2009. Devonian climate and reef evolution: Insights from oxygen isotopes in apatite. Earth Planet Sci Lett, 284: 599-609

Joachimski M M, Pancost R D, Freeman K H, Ostertag-Henning C, Buggisch W. 2002. Carbon isotope geochemistry of the FrasnianFamennian transition. Palaeogeogr Palaeoclimatol Palaeoecol, 181: 91-109 
Jones T D, Lunt D J, Schmidt D N, Ridgwell A, Sluijs A, Valdes P J, Maslin M. 2013. Climate model and proxy data constraints on ocean warming across the Paleocene-Eocene Thermal Maximum. EarthSci Rev, 125: 123-145

Jourdan F, Hodges K, Sell B, Schaltegger U, Wingate M T D, Evins L Z, Söderlund U, Haines P W, Phillips D, Blenkinsop T. 2014. Highprecision dating of the Kalkarindji large igneous province, Australia, and synchrony with the Early-Middle Cambrian (Stage 4-5) extinction. Geology, 42: 543-546

Kaiho K, Takeda K, Petrizzo M R, Zachos J C. 2006. Anomalous shifts in tropical Pacific planktonic and benthic foraminiferal test size during the Paleocene-Eocene thermal Maximum. Palaeogeogr Palaeoclimatol Palaeoecol, 237: 456-464

Kemp D B, Coe A L, Cohen A S, Schwark L. 2005. Astronomical pacing of methane release in the Early Jurassic period. Nature, 437: 396-399

Kemp D B, Coe A L, Cohen A S, Weedon G P. 2011. Astronomical forcing and chronology of the early Toarcian (Early Jurassic) oceanic anoxic event in Yorkshire, UK. Paleoceanography, 26: PA002122

Kennett J P, Stott L D. 1991. Abrupt deep-sea warming, palaeoceanographic changes and benthic extinctions at the end of the Palaeocene. Nature, 353: 225-229

Korbar T, Glumac B, Tesovic B C, Cadieux S B. 2012. Response of a carbonate platform to the Cenomanian-Turonian Drowning and OAE 2: A case study from the Adriatic Platform (Dalmatia, Croatia). J Sediment Res, 82: 163-176

Korte C, Hesselbo S P, Ullmann C V, Dietl G, Ruhl M, Schweigert G, Thibault N. 2015. Jurassic climate mode governed by ocean gateway. Nat Commun, 6: 10015

Kraus M J, Riggins S. 2007. Transient drying during the PaleoceneEocene Thermal Maximum (PETM): Analysis of paleosols in the Bighorn Basin, Wyoming. Palaeogeogr Palaeoclimatol Palaeoecol, 245: 444-461

Kraus M J, Woody D T, Smith J J, Dukic V. 2015. Alluvial response to the Paleocene-Eocene Thermal Maximum climatic event, Polecat Bench, Wyoming (U.S.A.). Palaeogeogr Palaeoclimatol Palaeoecol, 435: $177-192$

Krencker F N, Bodin S, Suan G, Heimhofer U, Kabiri L, Immenhauser A. 2015. Toarcian extreme warmth led to tropical cyclone intensification. Earth Planet Sci Lett, 425: 120-130

Krencker F N, Lindström S, Bodin S. 2019. A Major sea-level drop briefly precedes the Toarcian oceanic anoxic event: Implication for Early Jurassic climate and carbon cycle. Sci Rep, 9: 12518

Leckie R M, Bralower T J, Cashman R. 2002. Oceanic anoxic events and plankton evolution: Biotic response to tectonic forcing during the mid-Cretaceous. Paleoceanography, 17: 13-1-13-29

Li J, Hu X, Garzanti E, BouDagher-Fadel M. 2017. Shallow-water carbonate responses to the Paleocene-Eocene thermal Maximum in the Tethyan Himalaya (southern Tibet): Tectonic and climatic implications. Palaeogeogr Palaeoclimatol Palaeoecol, 466: 153-165

Li J, Hu X, Zhao K, Cai Y, Sun T. 2016. Paleoceanographic evolution and chronostratigraphy of the Aptian Oceanic Anoxic Event 1a (OAE1a) to oceanic red bed 1 (ORB1) in the Gorgo a Cerbara section (central Italy). Cretac Res, 66: 115-128

Li Y X, Bralower T J, Montañez I P, Osleger D A, Arthur M A, Bice D M, Herbert T D, Erba E, Premoli Silva I. 2008. Toward an orbital chronology for the early Aptian Oceanic Anoxic Event (OAE1a, $\sim 120$ Ma). Earth Planet Sci Lett, 271: 88-100

Li Y X, Montañez I P, Liu Z, Ma L. 2017. Astronomical constraints on global carbon-cycle perturbation during Oceanic Anoxic Event 2 (OAE2). Earth Planet Sci Lett, 462: 35-46

Ludvigson G A, Joeckel R M, Gonzalez L A, Gulbranson E L, Rasbury E T, Hunt G J, Kirkland J I, Madsen S. 2010. Correlation of AptianAlbian carbon isotope excursions in continental strata of the Cretaceous foreland basin, Eastern Utah, U.S.A. J Sediment Res, 80: 955-974

MacLeod K G, Martin E E, Blair S W. 2008. Nd isotopic excursion across Cretaceous ocean anoxic event 2 (Cenomanian-Turonian) in the tropical North Atlantic. Geology, 36: 811-814

McArthur J M, Donovan D T, Thirlwall M F, Fouke B W, Mattey D. 2000. Strontium isotope profile of the early Toarcian (Jurassic) oceanic anoxic event, the duration of ammonite biozones, and belemnite palaeotemperatures. Earth Planet Sci Lett, 179: 269-285

McElwain J C, Wade-Murphy J, Hesselbo S P. 2005. Changes in carbon dioxide during an oceanic anoxic event linked to intrusion into Gondwana coals. Nature, 435: 479-482

McInerney F A, Wing S L. 2011. The Paleocene-Eocene Thermal Maximum: A perturbation of carbon cycle, climate, and biosphere with implications for the future. Annu Rev Earth Planet Sci, 39: 489-516

Menegatti A P, Weissert H, Brown R S, Tyson R V, Farrimond P, Strasser A, Caron M. 1998. High-resolution $\delta^{13} \mathrm{C}$ stratigraphy through the Early Aptian "Livello selli" of the Alpine tethys. Paleoceanography, 13: 530-545

Montañez I P, Osleger D A, Banner J L. 2000. Evolution of the Sr and C isotope composition of Cambrian oceans. GSA today, 10: 1-7

Morten S D, Twitchett R J. 2009. Fluctuations in the body size of Marine invertebrates through the Pliensbachian-Toarcian extinction event. Palaeogeogr Palaeoclimatol Palaeoecol, 284: 29-38

Mülayim O, Yilmaz O I, Sarı B, Taslı K, Wagreich M. 2019. Cenomanian-Turonian drowning of the Arabian Carbonate Platform, 
the İnişdere section, Adıyaman, SE Turkey. Geol Soc Lond, 498: SP498-2018-130

Murphy A E, Sageman B B, Hollander D J, Lyons T W, Brett C E. 2000. Black shale deposition and faunal overturn in the Devonian Appalachian Basin: Clastic starvation, seasonal water-column mixing, and efficient biolimiting nutrient recycling. Paleoceanography, 15: 280-291

Murphy B H, Farley K A, Zachos J C. 2010. An extraterrestrial ${ }^{3} \mathrm{He}-$ based timescale for the Paleocene-Eocene thermal Maximum (PETM) from Walvis Ridge, IODP Site 1266. Geochim Cosmochim Acta, 74: 5098-5108

Mutterlose J, Bottini C, Schouten S, Sinninghe Damsté J S. 2014. High sea-surface temperatures during the early Aptian Oceanic Anoxic Event 1a in the Boreal Realm. Geology, 42: 439-442

Mutterlose J, Malkoc M, Schouten S, Sinninghe Damsté J S, Forster A. 2010. $\mathrm{TEX}_{86}$ and stable $\delta^{18} \mathrm{O}$ paleothermometry of early Cretaceous sediments: Implications for belemnite ecology and paleotemperature proxy application. Earth Planet Sci Lett, 298: 286-298

Naafs B D A, Pancost R D. 2016. Sea-surface temperature evolution across Aptian Oceanic Anoxic Event 1a. Geology, 44: 959-962

National Research Council (NRC). 2011. Understanding Earth's Deep Past: Lessons for Our Climate Future. Washington DC: The National Academies Press. 208

O’Brien C L, Robinson S A, Pancost R D, Sinninghe Damsté J S, Schouten S, Lunt D J, Alsenz H, Bornemann A, Bottini C, Brassell S C, Farnsworth A, Forster A, Huberl B T, Inglis G N, Jenkyns H C, Linnert C, Littler, K, Markwick P, McAnena A, Mutterlose J, Naafs, B D A, Püttmann W, Sluijs A, van Helmond N A G M, Vellekoop J, Wagner T, Wrobel N E. 2017. Cretaceous sea-surface temperature evolution: Constraints from $\mathrm{TEX}_{86}$ and planktonic foraminiferal oxygen isotopes. Earth-Sci Rev, 172: 224-247

Ogg J G, Ogg G M, Gradstein F M. 2016. A Concise Geologic Time Scale: 2016. Amsterdam: Elsevier. 240

Pälike C, Delaney M L, Zachos J C. 2014. Deep-sea redox across the Paleocene-Eocene thermal Maximum. Geochem Geophys Geosyst, 15: $1038-1053$

Pancost R D, Crawford N, Magness S, Turner A, Jenkyns H C, Maxwell J R. 2004. Further evidence for the development of photic-zone euxinic conditions during Mesozoic oceanic anoxic events. J Geol Soc, 161: 353-364

Patruno S, Triantaphyllou M V, Erba E, Dimiza M D, Bottini C, Kaminski M A. 2015. The Barremian and Aptian stepwise development of the 'Oceanic Anoxic Event 1a' (OAE 1a) crisis: Integrated benthic and planktic high-resolution palaeoecology along the Gorgo a Cerbara stratotype section (Umbria-Marche Basin, Italy). Palaeogeogr Palaeoclimatol Palaeoecol, 424: 147-182
Payne J L, Turchyn A V, Paytan A, Depaolo D J, Lehrmann D J, Yu M, Wei J. 2010. Calcium isotope constraints on the end-Permian Mass extinction. Proc Natl Acad Sci USA, 107: 8543-8548

Pearson P N, Nicholas C J. 2014. Layering in the Paleocene/Eocene boundary of the Millville core is drilling disturbance. Proc Natl Acad Sci USA, 111: E1064-E1065

Penman D E, Hönisch B, Zeebe R E, Thomas E, Zachos J C. 2014. Rapid and sustained surface ocean acidification during the Paleocene-Eocene Thermal Maximum. Paleoceanography, 29: 357-369

Percival L M E, Jenkyns H C, Mather T A, Dickson A J, Batenburg S J, Ruhl M, Hesselbo S P, Barclay R, Jarvis I, Robinson S A, Woelders L. 2018. Does large igneous province volcanism always perturb the mercury cycle? Comparing the records of Oceanic Anoxic Event 2 and the end-Cretaceous to other Mesozoic events. Am J Sci, 318: 799-860

Pogge von Strandmann P A E, Jenkyns H C, Woodfine R G. 2013. Lithium isotope evidence for enhanced weathering during Oceanic Anoxic Event 2. Nat Geosci, 6: 668-672

Pujalte V, Schmitz B, Baceta J I. 2014. Sea-level changes across the Paleocene-Eocene interval in the Spanish Pyrenees, and their possible relationship with North Atlantic Magmatism. Palaeogeogr Palaeoclimatol Palaeoecol, 393: 45-60

Racki G, Rakociński M, Marynowski L, Wignall P B. 2018. Mercury enrichments and the Frasnian-Famennian biotic crisis: A volcanic trigger proved? Geology, 46: 543-546

Röhl U, Norris R, Ogg J G. 2003. Cyclostratigraphy of upper Paleocene and lower Eocene sediments at Blake Nose Site 1051 (western North Atlantic). Geol Soc Am Spec Pap, 369: 567-588

Röhl U, Westerhold T, Bralower T J, Zachos J C. 2007. On the duration of the Paleocene-Eocene thermal Maximum (PETM). Geochem Geophys Geosyst, 8: Q12002

Robinson S A, Heimhofer U, Hesselbo S P, Petrizzo M R. 2017 Mesozoic climates and oceans: A tribute to Hugh Jenkyns and Helmut Weissert. Sedimentology, 64: 1-15

Ruebsam W, Münzberger P, Schwark L. 2014. Chronology of the early Toarcian environmental crisis in the Lorraine Sub-Basin (NE Paris Basin). Earth Planet Sci Lett, 404: 273-282

Sabatino N, Vlahović I, Jenkyns H C, Scopelliti G, Neri R, Prtoljan B, Velić I. 2013. Carbon-isotope record and palaeoenvironmental changes during the early Toarcian oceanic anoxic event in shallowmarine carbonates of the Adriatic Carbonate Platform in Croatia. Geol Mag, 150: 1085-1102

Sageman B B, Meyers S R, Arthur M A. 2006. Orbital time scale and new $\mathrm{C}$-isotope record for Cenomanian-Turonian boundary stratotype. Geology, 34: 125-128 
Scheibner C, Speijer R P, Marzouk A M. 2005. Turnover of larger foraminifera during the Paleocene-Eocene Thermal Maximum and paleoclimatic control on the evolution of platform ecosystems. Geology, 33: 493-496

Scheibner C, Speijer R P. 2008. Decline of coral reefs during late Paleocene to early Eocene global warming. EEarth, 3: 19-26

Schmitz B, Pujalte V. 2003. Sea-level, humidity, and land-erosion records across the initial Eocene thermal Maximum from a continental-marine transect in northern Spain. Geology, 31: 689692

Schmitz B, Pujalte V. 2007. Abrupt increase in seasonal extreme precipitation at the Paleocene-Eocene boundary. Geology, 35: 215218

Sepkoski Jr J J. 1996. Patterns of Phanerozoic extinction: A perspective from global data bases, Global events and event stratigraphy in the Phanerozoic. In: Walliser O, H, ed. Global Events and Event Stratigraphy in the Phanerozoic. Berlin: Springer-Verlag. 35-51

Shen S, Crowley J L, Wang Y, Bowring S A, Erwin D H, Sadler P M, Cao C, Rothman D H, Henderson C M, Ramezani J, Zhang H, Shen Y, Wang X, Wang W, Mu L, Li W, Tang Y, Liu X, Liu L, Zeng Y, Jiang Y, Jin Y. 2011. Calibrating the end-Permian Mass extinction. Science, 334: 1367-1372

Shen S Z, Ramezani J, Chen J, Cao C Q, Erwin D H, Zhang H, Xiang L, Schoepfer S D, Henderson C M, Zheng Q F, Bowring S A, Wang Y, Li X H, Wang X D, Yuan D X, Zhang Y C, Mu L, Wang J, Wu Y S. 2019. A sudden end-Permian Mass extinction in South China. GSA Bull, 131: 205-223

Simms M J, Ruffell A H. 1989. Synchroneity of climatic change and extinctions in the Late Triassic. Geology, 17: 265-268

Sinninghe Damsté J S, van Bentum E C, Reichart G J, Pross J, Schouten S. 2010. A $\mathrm{AO}_{2}$ decrease-driven cooling and increased latitudinal temperature gradient during the mid-Cretaceous Oceanic Anoxic Event 2. Earth Planet Sci Lett, 293: 97-103

Skelton P W, Gili E. 2012. Rudists and carbonate platforms in the Aptian: A case study on biotic interactions with ocean chemistry and climate. Sedimentology, 59: 81-117

Sluijs A, Bijl P K, Schouten S, Röhl U, Reichart G J, Brinkhuis H. 2011. Southern ocean warming, sea level and hydrological change during the Paleocene-Eocene thermal Maximum. Clim Past, 7: 4761

Sluijs A, Brinkhuis H. 2009. A dynamic climate and ecosystem state during the Paleocene-Eocene Thermal Maximum: Inferences from dinoflagellate cyst assemblages on the New Jersey Shelf. Biogeosciences, 6: 1755-1781

Sluijs A, Röhl U, Schouten S, Brumsack H J, Sangiorgi F, Sinninghe
Damsté J S, Brinkhuis H. 2008. Arctic late Paleocene-early Eocene paleoenvironments with special emphasis on the Paleocene-Eocene thermal Maximum (Lomonosov Ridge, Integrated Ocean Drilling Program Expedition 302). Paleoceanography, 23: PA1S11

Sluijs A, Schouten S, Pagani M, Woltering M, Brinkhuis H, Sinninghe Damsté J S, Dickens G R, Huber M, Reichart G J, Stein R, Matthiessen J, Lourens L J, Pedentchouk N, Backman J, Moran K. 2006. Subtropical Arctic Ocean temperatures during the Palaeocene/ Eocene Thermal Maximum. Nature, 441: 610-613

Sluijs A, van Roij L, Harrington G J, Schouten S, Sessa J A, LeVay L J, Reichart G J, Slomp C P. 2014. Warming, euxinia and sea level rise during the Paleocene-Eocene Thermal Maximum on the Gulf Coastal Plain: Implications for ocean oxygenation and nutrient cycling. Clim Past, 10: 1421-1439

Socorro J, Maurrasse F J M R, Sanchez-Hernandez Y. 2017. Characterization of the negative carbon isotope shift in segment $\mathrm{C}_{2}$, its global implications as a harbinger of OAE1a. Sci China Earth Sci, 60: 30-43

Song H, Wignall P B, Tong J, Yin H. 2013. Two pulses of extinction during the Permian-Triassic crisis. Nat Geosci, 6: 52-56

Speijer R P, Wagner T. 2002. Sea-level changes and black shales associated with the late Paleocene thermal Maximum (LPTM): Organic geochemical and micropaleontologic evidence from the southern Tethyan Margin (Egypt-Israel). Geol Soc Amer Spec Pap, 356: $522-550$

Stanley G D. 1989. An Upper Triassic reefal limestone, Southern Vancouver Island, B.C. In: Geldsetzer H H J, James N P, Tebbutt G E, eds. Reef: Canada and Adjacent Areas. Canadian Society of Petroleum Geologists. Memoirs, 13: 766-775

Stein M, Westermann S, Adatte T, Matera V, Fleitmann D, Spangenberg J E, Föllmi K B. 2012. Late Barremian-Early Aptian palaeoenvironmental change: The Cassis-La Bédoule section, southeast France. Cretac Res, 37: 209-222

Suan G, Mattioli E, Pittet B, Mailliot S, Lécuyer C. 2008a. Evidence for Major environmental perturbation prior to and during the Toarcian (Early Jurassic) oceanic anoxic event from the Lusitanian Basin, Portugal. Paleoceanography, 23: PA001202

Suan G, Pittet B, Bour I, Mattioli E, Duarte L, Mailliot S. 2008 b. Duration of the Early Toarcian carbon isotope excursion deduced from spectral analysis: Consequence for its possible causes. Earth Planet Sci Lett, 267: 666-679

Suan G, Mattioli E, Pittet B, Lécuyer C, Suchéras-Marx B, Duarte L V, Philippe M, Reggiani L, Martineau F. 2010. Secular environmental precursors to Early Toarcian (Jurassic) extreme climate changes. Earth Planet Sci Lett, 290: 448-458

Suarez M B, Ludvigson G A, González L A, You H L. 2017. 
Continental paleotemperatures from an early Cretaceous dolomitic lake, Gansu province, China. J Sediment Res, 87: 486-499

Sun Y, Joachimski M M, Wignall P B, Yan C, Chen Y, Jiang H, Wang L, Lai X. 2012. Lethally hot temperatures during the early Triassic Greenhouse. Science, 338: 366-370

Sun Y D, Richoz S, Krystyn L, Zhang Z T, Joachimski M M. 2019. Perturbations in the carbon cycle during the Carnian Humid Episode: Carbonate carbon isotope records from southwestern China and northern Oman. J Geol Soc, 176: 167-177

Takeda K, Kaiho K. 2007. Faunal turnovers in central Pacific benthic foraminifera during the Paleocene-Eocene thermal Maximum. Palaeogeogr Palaeoclimatol Palaeoecol, 251: 175-197

Thomas D J, Zachos J C, Bralower T J, Thomas E, Bohaty S. 2002. Warming the fuel for the fire: Evidence for the thermal dissociation of methane hydrate during the Paleocene-Eocene thermal Maximum. Geology, 30: 1067-1070

Trecalli A, Spangenberg J, Adatte T, Föllmi K B, Parente M. 2012. Carbonate platform evidence of ocean acidification at the onset of the early Toarcian oceanic anoxic event. Earth Planet Sci Lett, 357: 214-225

Turner S K. 2018. Constraints on the onset duration of the PaleoceneEocene Thermal Maximum. Phil Trans R Soc A, 376: 20170082 van Bentum E C, Hetzel A, Brumsack H J, Forster A, Reichart G J, Sinninghe Damsté J S. 2009. Reconstruction of water column anoxia in the equatorial Atlantic during the Cenomanian-Turonian oceanic anoxic event using biomarker and trace metal proxies. Palaeogeogr Palaeoclimatol Palaeoecol, 280: 489-498

van Helmond N A G M, Ruvalcaba Baroni I, Sluijs A, Sinninghe Damsté J S, Slomp C P. 2014. Spatial extent and degree of oxygen depletion in the deep proto-North Atlantic basin during Oceanic Anoxic Event 2. Geochem Geophys Geosyst, 15: 4254-4266

Ward P D, Botha J, Buick R, De Kock M O, Erwin D H, Garrison G H, Kirschvink J L, Smith R. 2005. Abrupt and gradual extinction among late Permian land vertebrates in the Karoo Basin, South Africa. Science, 307: 709-714

Ward P D, Montgomery D R, Smith R. 2000. Altered river morphology in South Africa related to the Permian-Triassic extinction. Science, 289: $1740-1743$

Waters C N, Zalasiewicz J, Summerhayes C, Barnosky A D, Poirier C, Gałuszka A, Cearreta A, Edgeworth M, Ellis E C, Ellis M, Jeandel C, Leinfelder R, McNeill J R, Richter D B, Steffen W, Syvitski J, Vidas D, Wagreich M, Williams M, Zhisheng A, Grinevald J, Odada E, Oreskes N, Wolfe A P. 2016. The Anthropocene is functionally and stratigraphically distinct from the Holocene. Science, 351: $\operatorname{aad} 2622$

Westerhold T, Röhl U, Donner B, Zachos J C. 2018. Global extent of early Eocene hyperthermal events: A new Pacific benthic foraminiferal isotope record from Shatsky Rise (ODP Site 1209). Paleoceanogr Paleoclimatol, 33: 626-642

Westermann S, Stein M, Matera V, Fiet N, Fleitmann D, Adatte T, Föllmi K B. 2013. Rapid changes in the redox conditions of the western Tethys Ocean during the early Aptian oceanic anoxic event. Geochim Cosmochim Acta, 121: 467-486

Wignall P B. 2001. Large igneous provinces and Mass extinctions. Earth-Sci Rev, 53: 1-33

Wilson E O, MacArthur R H. 1967. The Theory of Island Biogeography. Princeton N J: Princeton University Press

Wing S L, Harrington G J, Smith F A, Bloch J I, Boyer D M, Freeman K H. 2005. Transient floral change and rapid global warming at the Paleocene-Eocene boundary. Science, 310: 993-996

Winguth A M E, Thomas E, Winguth C. 2012. Global decline in ocean ventilation, oxygenation, and productivity during the PaleoceneEocene Thermal Maximum: Implications for the benthic extinction. Geology, 40: 263-266

Wright J D, Schaller M F. 2013. Evidence for a rapid release of carbon at the Paleocene-Eocene thermal Maximum. Proc Natl Acad Sci USA, 110: 15908-15913

Xu B, Gu Z, Wang C, Hao Q, Han J, Liu Q, Wang L, Lu Y. 2012. Carbon isotopic evidence for the associations of decreasing atmospheric $\mathrm{CO}_{2}$ level with the Frasnian-Famennian Mass extinction. J Geophys Res, 170: 1-12

Yin H, Jiang H, Xia W, Feng Q, Zhang N, Shen J. 2014. The endPermian regression in South China and its implication on Mass extinction. Earth-Sci Rev, 137: 19-33

Yu J, Broutin J, Chen Z Q, Shi X, Li H, Chu D, Huang Q. 2015. Vegetation changeover across the Permian-Triassic boundary in southwest China. Earth-Sci Rev, 149: 203-224

Zachos J C, Röhl U, Schellenberg S A, Sluijs A, Hodell D A, Kelly D C, Thomas E, Nicolo M, Raffi I, Lourens L J, McCarren H, Kroon D. 2005. Rapid acidification of the ocean during the PaleoceneEocene Thermal Maximum. Science, 308: 1611-1615

Zachos J C, Schouten S, Bohaty S, Quattlebaum T, Sluijs A, Brinkhuis H, Gibbs S J, Bralower T J. 2006. Extreme warming of mid-latitude coastal ocean during the Paleocene-Eocene Thermal Maximum: Inferences from $\mathrm{TEX}_{86}$ and isotope data. Geology, 34: 737-740

Zachos J C, Wara M W, Bohaty S, Delaney M L, Petrizzo M R, Brill A, Bralower T J, Premoli-Silva I. 2003. A transient rise in tropical sea surface temperature during the Paleocene-Eocene thermal Maximum. Science, 302: 1551-1554

Zachos J, Pagani M, Sloan L, Thomas E, Billups K. 2001. Trends, rhythms, and aberrations in global climate $65 \mathrm{Ma}$ to present. Science, 292: 686-693 
Zeebe R E, Dickens G R, Ridgwell A, Sluijs A, Thomas E. 2014. Onset of carbon isotope excursion at the Paleocene-Eocene thermal Maximum took millennia, not 13 years. Proc Natl Acad Sci USA, 111: E1062-E1063

Zeebe R E, Zachos J C, Dickens G R. 2009. Carbon dioxide forcing alone insufficient to explain Palaeocene-Eocene Thermal Maximum warming. Nat Geosci, 2: 576-580

Zheng X Y, Jenkyns H C, Gale A S, Ward D J, Henderson G M. 2013. Changing ocean circulation and hydrothermal inputs during Ocean
Anoxic Event 2 (Cenomanian-Turonian): Evidence from Ndisotopes in the European shelf sea. Earth Planet Sci Lett, 375: 338-348

Zhu Z, Liu Y, Kuang H, Benton M J, Newell A J, Xu H, An W, Ji S ', Xu S, Peng N, Zhai Q. 2019. Altered fluvial patterns in North China indicate rapid climate change linked to the Permian-Triassic Mass extinction. Sci Rep, 9: 16818

Zhuravlev A Y, Wood R A. 1996. Anoxia as the cause of the mid-Early Cambrian (Botomian) extinction event. Geology, 24: 311-314

(责任编委: 胡建芳) 